\title{
MODELAGEM NUMÉRICA DA COMPOSIÇÃO QUÍMICA DA ATMOSFERA E SEUS IMPACTOS NO TEMPO, CLIMA E QUALIDADE DO AR
}

\author{
SAULO R. FREITAS ${ }^{1}$, KARLA M. LONGO ${ }^{2}$, LUIZ F. RODRIGUES ${ }^{1}$ \\ ${ }^{1}$ Centro de Previsão de Tempo e Estudos Climáticos, Instituto Nacional de \\ Pesquisas Espaciais (CPTEC/INPE), Cachoeira Paulista, Brasil. \\ ${ }^{2}$ Divisão de Geofísica Espacial, Instituto Nacional de Pesquisas Espaciais (DGE/INPE) \\ saulo.freitas@cptec.inpe.br, karla.longo@dge.inpe.br, luiz.flavio@cptec.inpe.br
}

Recebido Julho 2008 - Aceito Março 2009

\begin{abstract}
RESUMO
Uma descrição geral da modelagem numérica da composição química da atmosfera é fornecida neste trabalho. Os papéis relevantes de alguns gases traço e aerossóis no balanço radiativo do planeta, na alteração do ciclo hidrológico e na poluição do ar são discutidos. Partindo da equação da continuidade, integrada no sistema de equações governantes da atmosfera, os diversos termos que constituem a emissão, deposição, reatividade química e transporte (resolvido e sub-grade) são apresentados juntamente com formas de obter a solução numérica. Dentre os componentes desta equação, o termo de reatividade química se destaca pela complexidade do problema em si, na obtenção da solução numérica e no alto custo computacional envolvido. Exemplos são fornecidos utilizando o modelo CCATT-BRAMS com recente inclusão de reatividade química. Em particular, a simulação do ozônio troposférico constitui uma tarefa complexa pela falta de dados de emissões, porém resultados obtidos são compatíveis com outros modelos validados. Simulações numéricas realizadas, para a estação seca de 2002, permitiram estudar possíveis ciclos biogeoquímicos e corredores de formação e deposição de ozônio e aerossóis de queimadas, assim como o papel destes últimos na estabilização termodinâmica e precipitação.

Palavras-chave: Qualidade do ar, gases traço, aerossóis, química da atmosfera, modelos ambientais.
\end{abstract}

\begin{abstract}
NUMERICAL MODELING OF THE ATMOSPHERE CHEMISTRY COMPOSITION AND OF ITS IMPACTS ON WEATHER, CLIMATE AND AIR QUALITY.

A general description of the numerical modeling of the atmospheric chemical composition is presented. The relevant role played by some trace gases and aerosols on the Earth radiative budget, hydrological cycle and air quality are discussed. The formulation for emission, deposition, chemical reactions and transport (resolved and sub-grid scale) processes are introduced within the mass continuity equation integrated to the basic equations system for the Earth atmosphere. The numerical aspects of this formulation are as well discussed, specially the intrinsic complexity and the high computational costs of the chemical reactions system. Examples are showed using results obtained with the CCATTBRAMS model, such as the sensitivity of the tropospheric ozone simulation to reliable emission data and comparison of the CCATT-BRAMS results with other model. Numerical simulations performed for the 2002 dry season allowed to study possible biogeochemical cycles and corridors of tropospheric ozone formation, transport and deposition from precursor's emissions by biomass burning activities. We also looked at the atmospheric model response to the direct effect of biomass burning aerosols on the thermodynamic equation and on the convective inhibition.
\end{abstract}

Keywords: Air quality, trace gases, aerosol, atmospheric chemistry, numerical modeling 


\section{INTRODUÇÃO}

As perturbações antrópicas no sistema Terrestre têm sido inequivocamente, associadas às mudanças climáticas observadas e lançam fundamentada preocupação quanto à sustentabilidade e vulnerabilidade deste para a sociedade humana no futuro (IPCC, 2007). Dentre as perturbações antrópicas, se destacam as emissões de gases do efeito estufa, de diversos tipos de aerossóis, gases primários tóxicos e/ou precursores de gases secundários nocivos à saúde, como por exemplo, o ozônio $\left(\mathrm{O}_{3}\right)$, e a mudança de uso e cobertura do solo.

As queimadas antropogênicas, que ocorrem majoritariamente em áreas tropicais do planeta, são fontes importantes de gases do efeito estufa, aerossóis e poluentes para a atmosfera (Andreae et al., 2004, Artaxo et al., 2002, Andreae, 1991). Na América do Sul, durante os meses de inverno, ocorrem centenas de milhares de focos de incêndio, principalmente em ecossistemas de cerrado e floresta, estando majoritariamente associados a práticas agrícolas. Estas queimadas ocorrem primariamente nas regiões Amazônica e do Brasil Central, porém, através do transporte atmosférico de suas emissões, produzem uma distribuição espacial de fumaça sobre uma extensa área, ao redor de 4-5 milhões de $\mathrm{km}^{2}$, em muito superior à área onde estão concentradas (Freitas et al., 1999, 2005, 2006, 2007B). Durante a combustão de biomassa, são emitidos para a atmosfera gases, incluindo alguns de efeito estufa e precursores do ozônio troposférico, e partículas de aerossol que interagem eficientemente com a radiação solar e afetam os processos de microfísica e dinâmica de formação de nuvens e a qualidade do ar. Os efeitos destas emissões excedem, portanto, a escala local e afetam regionalmente a composição e propriedades físicas e químicas da atmosfera na América do Sul e áreas oceânicas vizinhas, com potencial impacto em escala global.

Em escalas regional e global, as emissões de queimadas alteram o balanço radiativo da atmosfera, através dos efeitos diretos das partículas de aerossóis ao refletir e espalhar a radiação solar de volta para o espaço, reduzindo a quantidade absorvida pela superfície terrestre, e ao absorver radiação solar, aquecendo a atmosfera. Jacobson (2001) sugere que o aquecimento atmosférico devido aos aerossóis do tipo 'black carbon' poderia balancear o efeito de resfriamento associado a outros tipos (sulfatos), e que a forçante radiativa direta destes pode exceder aquele associado ao $\mathrm{CH}_{4}$. Desta forma, partículas de aerossol, produto de processos de combustão incompleta, estariam atrás somente do $\mathrm{CO}_{2}$ na contribuição para o aquecimento radiativo da atmosfera. Recentemente, Cox et al. (2008) apresentaram um novo aspecto do papel dos aerossóis reflexivos (sulfatos) sobre o clima. Ao contrabalançarem o efeito dos gases do efeito estufa, reduzem o aquecimento do Oceano
Atlântico e, assim, diminuem o risco de secas na Amazônia, tal qual a de 2005 (Marengo et al., 2008).

O balanço de radiação e o ciclo hidrológico, também podem ser afetados indiretamente pelas emissões de queimadas via alterações na microfísica e na dinâmica de formação de nuvens (Kaufman, 1995), em função de uma maior disponibilidade de núcleos de condensação de nuvem (NCN) e gelo na atmosfera, os quais promovem mudanças no espectro de gotas de nuvem (Andreae et al., 2004; Koren et al., 2004, Rosenfeld, 1999) e estabilização termodinâmica (Longo et al., 2006). O aumento da concentração de partículas de aerossol impõe a produção de gotículas de nuvem menores e em maior número, produzindo dois efeitos: primeiro, a maior quantidade de gotículas reflete mais radiação solar de volta para o espaço (portanto resfriando a atmosfera), e, segundo, o tamanho menor será menos favorável para a produção de chuva, pois gotículas muito pequenas não tendem a se aglutinarem para formar as grandes gotas que caem como chuva. Por outro lado, a estabilização termodinâmica imposta pela interação direta das partículas de aerossol com a radiação solar (redução do aquecimento na baixa atmosfera por redução da radiação solar) restringe a ascensão de células convectivas geradas próximo à superfície e, assim, inibe a formação de nuvens. Este conjunto de fatores sugere que os efeitos das queimadas podem extrapolar a escala local e afetar, de maneira importante, o ciclo hidrológico numa escala regional e o padrão da redistribuição planetária de energia dos trópicos para as latitudes médias e altas.

As mudanças da composição química da atmosfera relativas à qualidade do ar, também são objeto de crescente preocupação em geral. As populações que vivem tanto nas megacidades do planeta, quanto em regiões de intensas queimadas, estão sujeitas a um crescente número de dias em que a qualidade do ar ultrapassa os níveis de qualidade recomendáveis pela Organização Mundial de Saúde, e previstos na legislação ambiental de diversos países. Dentre os poluentes com maior impacto na saúde pública, se destacam o material particulado fino (PM2.5) e o $\mathrm{O}_{3}$. O ozônio é altamente tóxico, implicando em efeitos na saúde humana, quando respirado, e também produz degradação de plantas presentes em regiões contaminadas. $\mathrm{O} \mathrm{O}_{3}$ é um poluente secundário não sendo emitido diretamente, e compõe o rol dos gases do efeito estufa mais importante. Ainda, seus precursores estão relacionados, principalmente às atividades de combustão e biogênicas. Um aspecto relevante é o de que o aquecimento global pode induzir uma situação ainda mais crítica, em termos de qualidade do ar, ao intensificar as taxas de reatividade química com o aumento das temperaturas próximas à superfície.

Por outro lado, mudanças no uso da terra provocam alterações no balanço de energia, água e de momentum na 
superfície devido às correspondentes mudanças no albedo da superfície, capacidade de evapotranspiração associada à cobertura vegetal e sua estrutura espacial. Em particular, a substituição de áreas florestadas com sistema radicular profundo por áreas de pastagens, implica em aumento no albedo e baixa acessibilidade a solos profundos com alto armazenamento de água. Esta mudança leva, em geral, a uma inversão na razão de Bowen, produzindo camadas limite planetárias mais secas, quentes e profundas, principalmente durante a estação seca. Desta forma, mudanças no uso da terra são por si só indutoras de alteração no padrão do ciclo hidrológico. Certamente, estas alterações afetam também o transporte e ciclos de gases traço. Outro aspecto relevante corresponde ao efeito da mudança do uso da terra nos aerossóis de poeira de solo. Com solos mais expostos e ventos mais intensos (o que se espera com a diminuição da rugosidade do terreno ao trocar florestas por pastagens) poderá haver um aumento significativo na produção e levantamento de poeira de solo com impactos também no balanço radiativo, microfísica de nuvens e ciclo hidrológico.

Todas estas questões urgem pelo desenvolvimento de modelos atmosféricos, nos quais as componentes de química e aerossóis atmosféricos estejam consistentemente incluídas. Modelos numéricos com solução do transporte, química e aerossóis atmosféricos acoplada à solução do estado atmosférico (on-line) representam o estado-da-arte em modelagem acoplada da atmosfera. A utilização de um sistema único de coordenadas vertical e horizontal, e das mesmas parametrizações físicas do modelo atmosférico para o transporte de elementos traço nas escalas de grade e sub-grade do modelo, faz com que o transporte atmosférico das partículas de aerossol e gases traço seja consistente com o modelo atmosférico em si, além de minimizar erros numéricos associados às interpolações em $(x, y, z, t)$, inevitáveis em modelos não acoplados (off-line), e que levam a violação da conservação de massa, dentre outros problemas. Além disto, modelos acoplados permitem a retro-alimentação do estado atmosférico com as perturbações decorrentes da presença de poluentes atmosféricos a cada passo no tempo do modelo. Esta abordagem segue a tendência geral de desenvolvimentos de modelos integrados, veja, por exemplo, o modelo WRF-CHEM (Weather Research and Forecasting model, http://wrf-model.org). Um exemplo interessante da importância da modelagem acoplada em escala climática é dado em Andreae et al. (2005). Neste trabalho os autores chamam a atenção para o extraordinário aumento na complexidade do cenário de mudanças climáticas a partir do reconhecimento da importância dos efeitos das partículas de aerossóis no clima. Até recentemente, os efeitos bem conhecidos dos gases de efeito estufa eram o personagem mais atuantes nos modelos de previsão climática. A inclusão dos aerossóis, nos modelos de tempo e clima, traz novos desafios em termos de desenvolvimentos de novas parametrizações que possam representar apropriadamente os diversos mecanismos através dos quais, essas interações ocorrem, gerando previsões confiáveis.

Este trabalho apresenta uma breve introdução à modelagem numérica da atmosfera incorporando gases traço e aerossóis, e apesar de não constituir uma descrição do sistema de modelagem denominado "Coupled Aerosol and Tracer Transport model to the Brazilian developments on the Regional Atmospheric Modeling System" (CATT-BRAMS, Freitas et al., 2005, 2007, Longo et al., 2006, 2007), utiliza-se de diversas parametrizações e resultados deste como exemplos desta modelagem.

\section{FORMULAÇÃO DOS MODELOS DE QUALIDADE DO AR}

\subsection{Sistema de equações governantes do estado atmosférico incluindo gases e aerossóis}

A atmosfera é considerada um sistema macroscópico e de baixa velocidade e, portanto, regido pelas leis da física clássica. No entanto, alguns processos atmosféricos, por exemplo, interação da radiação com os gases e reatividade química, necessitam da mecânica quântica ou da denominada física semiclássica para uma descrição precisa. O sistema de Equações 1,

$$
\left\{\begin{array}{l}
\frac{\partial \overrightarrow{\boldsymbol{v}}}{\partial \boldsymbol{t}}=-\overrightarrow{\boldsymbol{v}} \cdot \nabla \overrightarrow{\boldsymbol{v}}-\frac{1}{\rho_{a}} \nabla \boldsymbol{p}-\boldsymbol{g} \overrightarrow{\boldsymbol{k}}-2 \vec{\Omega} \times \overrightarrow{\boldsymbol{v}}+\overrightarrow{\boldsymbol{F}}_{\boldsymbol{v} i s c} \\
\frac{\partial \rho_{a}}{\partial \boldsymbol{t}}=-\nabla \cdot \rho_{a} \overrightarrow{\boldsymbol{v}} \\
\frac{\partial \theta}{\partial \boldsymbol{t}}=-\overrightarrow{\boldsymbol{v}} \cdot \nabla \theta+\boldsymbol{Q}_{\theta} \\
\frac{\partial \boldsymbol{r}_{n}}{\partial \boldsymbol{t}}=-\overrightarrow{\boldsymbol{v}} \cdot \nabla \boldsymbol{r}_{n}+\boldsymbol{Q}_{r_{n}} \\
\frac{\partial \boldsymbol{s}_{[\eta]}}{\partial \boldsymbol{t}}=-\overrightarrow{\boldsymbol{v}} \cdot \nabla \boldsymbol{s}_{[\eta]}+\boldsymbol{Q}_{s_{[]]}}
\end{array}\right.
$$

juntamente com a equação de gás ideal, é assumido governar a evolução da atmosfera, sendo seu estado futuro podendo ser determinado a partir do conhecimento do seu estado inicial:

A primeira Equação 1.1 estabelece a evolução temporal da velocidade do fluido atmosférico $(\overrightarrow{\boldsymbol{v}})$ em função do gradiente de pressão (p), da força gravitacional, da força de Coriolis (referencial terrestre é não inercial) e da viscosidade, numa descrição Euleriana. Esta é a lei de movimento de Newton. A Equação 1.2 expressa matematicamente a conservação de massa, 
ou equação da continuidade, descrita em termos de densidade de massa $\left(\rho_{\mathrm{a}}\right)$. A Equação 1.3 é a $1^{\mathrm{a}}$ lei da termodinâmica expressa em termos da temperatura potencial $(\theta)$ com o termo $\boldsymbol{Q}_{\theta}$, representando genericamente processos diabáticos (como exemplos, convergência de radiação e mudança de fase da água). A Equação 1.4 expressa matematicamente a conservação de massa de água em todas as fases existentes, denotadas pelo índice $\boldsymbol{n}$, em termos de razão de mistura de massa $\left(\rho_{\boldsymbol{n}} / \rho_{\boldsymbol{a}}\right) \cdot \boldsymbol{Q} \boldsymbol{r}_{\boldsymbol{n}}$ representa os termos fontes (evapotranspiração da superfície, mudanças de fase, etc.) e sumidouros (precipitação, mudança de fase, etc.) para as diversas formas e fases da água na atmosfera. A Equação 1.5 é a equação de continuidade de espécies gasosas e aerossóis presentes na atmosfera. Neste caso, $\boldsymbol{Q} \boldsymbol{s}_{|\boldsymbol{n}|}$ representa matematicamente um complexo conjunto de processos químicos e físicos que atuam na produção ou perda de massa (como exemplos, emissão, deposição seca e úmida, fotodissociação, reações químicas, sedimentação gravitacional, etc.). Em escalas de tempo e numa atmosfera não poluída, geralmente, apenas as Equações 1.1 a 1.4 são utilizadas para a determinação da evolução da atmosfera. Embora, de algum modo, a informação do conteúdo de aerossóis relevantes para o processo de microfísica de nuvens e a distribuição dos gases importantes para a transferência radiativa são incluídos. Porém, estes processos são tratados estaticamente, sem evolução temporal e com distribuição espacial homogênea. Em regiões com intensas fontes poluidoras, como na Amazônia e região central do Brasil na estação de queima, o efeito dos aerossóis de queimadas se faz importante no aspecto radiativo e de microfísica de nuvens, mesmo na escala de tempo e a Equação 1.5 precisa ser incluída na solução do sistema. Neste caso, as contribuições dos aerossóis na dinâmica da atmosfera são incorporadas nos forçantes $\boldsymbol{Q}_{\theta} \mathrm{e}$ $Q \boldsymbol{r}_{\boldsymbol{n}}$, respectivamente.

No moderno conceito de previsão ambiental, a qualidade do ar é um item tão importante quanto a previsão do tempo meteorológico. Neste contexto, a solução da Equação 1.5 é incluída na produção operacional dos centros de previsão de tempo e produtos gráficos com indicadores relacionados à criticidade para a saúde pública são disponibilizados.

Em problemas reais, o sistema de Equações 1 não possui solução analítica. Isto impõe a busca de solução numérica através de alguma metodologia de discretização (por exemplo, diferenças finitas). Obviamente, a solução mais perfeita seria a forma analítica que definiria o estado atmosférico continuamente no espaço-tempo. A discretização impõe solução em pontos específicos, distribuídos numa grade, do espaço-tempo e separa todas as escalas de movimento atmosféricos possíveis em duas famílias: resolvidas e não-resolvidas, dependendo dos espaçamentos da grade e intervalos de tempo de integração adotados. Além disto, a solução passa a representar uma média estatística de cada variável prognóstica dentro de cada célula do espaço-tempo discretizado. A decomposição de Reynolds é largamente utilizada para a solução numérica do sistema de Equações 1. Neste procedimento, cada variável (s) é separada em termos da média ( $\overline{\boldsymbol{s}})$ e da flutuação em torno desta $\left(\boldsymbol{s}^{\prime}\right)$ :

$$
s=\bar{s}+s^{\prime}
$$

com o termo $(\overline{\boldsymbol{s}})$ representando a média de volume e no intervalo de tempo considerado. Aplicando o procedimento de Reynolds à equação de continuidade, obtêm-se:

$$
\frac{\partial \overline{\boldsymbol{s}}}{\partial \boldsymbol{t}}+\underbrace{\overline{\boldsymbol{u}} \frac{\partial \overline{\boldsymbol{s}}}{\partial \boldsymbol{x}}+\overline{\boldsymbol{v}} \frac{\partial \overline{\boldsymbol{s}}}{\partial \boldsymbol{y}}+\overline{\boldsymbol{w}} \frac{\partial \overline{\boldsymbol{s}}}{\partial \boldsymbol{z}}}_{\text {I }}=\underbrace{-\frac{1}{\rho_{0}}\left(\frac{\partial \rho_{0} \overline{\boldsymbol{u}^{\prime} \boldsymbol{s}^{\prime}}}{\partial \boldsymbol{x}}+\frac{\partial \rho_{0} \overline{\boldsymbol{v}^{\prime} \boldsymbol{s}^{\prime}}}{\partial \boldsymbol{y}}+\frac{\partial \rho_{0} \overline{\boldsymbol{w}^{\prime} \boldsymbol{s}^{\prime}}}{\partial \boldsymbol{z}}\right)}_{\text {II }}+\overline{\boldsymbol{Q}_{s}}
$$

onde o termo I representa o transporte resolvido (advecção na escala da grade) e o termo II a contribuição do transporte nãoresolvido (transporte sub-grade por turbulência, convecção, etc.). O termo $\rho_{0}$ representa a densidade do ar no estado básico a partir da qual as flutuações são calculadas. Devido à nãolinearidade das equações, o efeito líquido dos fluxos turbulentos $\left(\overline{u_{i}^{\prime} \boldsymbol{s}^{\prime}}\right)$ é não nulo e o transporte sub-grade precisa ser incluído da solução da Equação 3. Definir os fluxos turbulentos em termos de variáveis da escala da grade é normalmente referido como parametrização e constitui um passo necessário para a solução da Equação 3. Uma vez definidos os fluxos turbulentos, conhecendo-se a relação funcional do termo forçante $\left(\overline{\boldsymbol{Q}_{s}}\right)$ e a condição inicial e de contorno, a Equação 3 pode, em princípio, ser resolvida. No entanto, a limitação computacional não nos permite a solução desta com todos os termos simultaneamente. Isto decorre do fato de que os termos de transporte acopla as três dimensões espaciais $(\boldsymbol{n} \boldsymbol{x}, \boldsymbol{n y}, \boldsymbol{n z})$ com as $\boldsymbol{n}$ espécies químicas gerando um sistema com $10^{4}$ ou mais equações. A técnica de separação por processos (splitting operator, Lanser e Verwer, 1998) é comumente utilizada para resolver cada processo independentemente e, então, agregar as mudanças resultantes de cada cálculo parcial para compor uma solução aproximada para a Equação 3. Desta forma a Equação 3 é aproximada pelo sistema abaixo:

$$
\left\{\begin{array}{l}
\left(\frac{\partial \bar{s}}{\partial \boldsymbol{t}}\right)_{a d v}=-\sum_{i} \bar{u}_{i} \frac{\partial \overline{\boldsymbol{s}}}{\partial \boldsymbol{x}_{i}} \\
\left(\frac{\partial \overline{\boldsymbol{s}}}{\partial \boldsymbol{t}}\right)_{\text {curb }}=-\frac{1}{\rho_{0}} \sum_{i} \frac{\partial \rho_{0}\left(\overline{\boldsymbol{u}_{i}^{\prime} \boldsymbol{s}^{\prime}}\right)_{\text {turb }}}{\partial \boldsymbol{x}_{\boldsymbol{i}}} \\
\left(\frac{\partial \overline{\boldsymbol{s}}}{\partial \boldsymbol{t}}\right)_{c o n v}=-\frac{1}{\rho_{0}} \sum_{i} \frac{\partial \rho_{0}\left(\overline{\boldsymbol{u}_{i}^{\prime} \boldsymbol{s}^{\prime}}\right)_{c o n v}}{\partial \boldsymbol{x}_{\boldsymbol{i}}} \\
\cdots \\
\left(\frac{\partial \overline{\boldsymbol{s}}}{\partial \boldsymbol{t}}\right)_{Q}=\overline{Q_{s}}
\end{array}\right.
$$


onde os índices adv, turb CLP e conv expressam os processos de advecção na escala da grade, turbulência dentro da camada limite planetária (CLP) e transporte convectivo por nuvens rasas e profundas não resolvidas explicitamente. Para obter a tendência total para o prognóstico final, cada tendência parcial é resolvida separadamente. A solução final pode ser aproximada compondo paralelamente ou seqüencialmente, este último na forma direta ou simétrica, as soluções parciais. No caso paralelo, cada equação parcial é resolvida a partir da mesma condição inicial associada ao começo do intervalo de tempo da integração, isto é, todos os processos são integrados a partir do mesmo valor inicial da razão de mistura. A solução final é então obtida a partir da soma das soluções parciais:

$$
\left(\frac{\partial \overline{\boldsymbol{s}}}{\partial t}\right)=\left(\frac{\partial \overline{\boldsymbol{s}}}{\partial \boldsymbol{t}}\right)_{a d v}+\left(\frac{\partial \overline{\boldsymbol{s}}}{\partial \boldsymbol{t}}\right)_{\text {turb }}+\left(\frac{\partial \overline{\boldsymbol{s}}}{\partial \boldsymbol{t}}\right)_{c o n v}+\ldots+\left(\frac{\partial \overline{\boldsymbol{s}}}{\partial \boldsymbol{t}}\right)_{Q}
$$

Uma vez obtida a tendência total, a razão de mistura é atualiza através da equação:

$$
\bar{s}(t+\Delta t)=\bar{s}(t)+\left(\frac{\partial \bar{s}}{\partial t}\right) \Delta t
$$

onde $\Delta t$ representa o intervalo de tempo da integração.

\subsection{Descrição dos termos de transporte}

Nesta seção, descreveremos os diversos termos de transporte envolvidos na Equação 3, seguindo o exemplo do modelo CATT-BRAMS.

O termo de transporte I da Equação 3 é resolvido numericamente por esquemas de advecção. Esquemas de advecção adequados devem conservar a massa, ser monotônicos, manter a solução positiva, ser local, eficiente do ponto de vista numérico e não demandar excessiva informação lateral para compor os gradientes necessários para a solução (o que requereria grande massa de dados trafegando pela rede em modelos paralelos com memória distribuída). No sistema CATTBRAMS advecção de escalares é resolvida com um esquema de $2^{\mathrm{a}}$ ordem e limitadores de fluxo para manter a solução positiva (Tremback et al., 1987) onde $\Delta \boldsymbol{x}_{\boldsymbol{i}}$ representa os espaçamentos de grade, e os fluxos $\boldsymbol{F}$ são avaliados nos pontos intermediários utilizando-se uma grade Arakawa-C. Trata-se se um esquema numericamente eficiente que necessita apenas das informações dos primeiros vizinhos. No entanto, em situação de fortes gradientes da quantidade advectada, não é estritamente positivo definido, gerando oscilações vento acima. Nestes casos, o esquema também apresenta significativa difusão numérica. Para estes esquemas, é necessária a inclusão de filtros que eliminem concentrações negativas, pois estas, mesmos pequenas, podem levar à instabilização numérica nos esquemas de reatividade química.

Para calcular o transporte na escala da sub-grade associado à difusão dentro da CLP, é necessária a determinação dos fluxos turbulentos $\overline{\boldsymbol{u}_{i}^{\prime} \boldsymbol{s}^{\prime}}$ Uma parametrização usual, denominada teoria $\mathrm{K}$ (veja, por exemplo, Stull, 1988), relaciona os fluxos turbulentos ao gradiente da quantidade média transportada por meio de um coeficiente de difusividade $(\boldsymbol{K})$

$$
\overline{u_{i}^{\prime} \boldsymbol{s}^{\prime}}=-\boldsymbol{K}_{h_{i}} \frac{\partial \overline{\boldsymbol{s}}}{\partial \boldsymbol{x}_{i}}
$$

Os coeficientes de difusividade são especificados em função das características da circulação (por exemplo, cisalhamento do vento, estabilidade termodinâmica, escalas de comprimento). Para ilustrar os diversos processos de transporte, resultados de diversas simulações numéricas reais executadas previamente serão utilizados. A Figura 1 apresenta o ciclo diurno da mistura turbulenta, através de uma simulação numérica sobre a região Amazônica. O painel A apresenta a evolução temporal do coeficiente de difusividade vertical. Durante uma situação convectiva, o coeficiente se intensifica ao longo do dia e a CLP se aprofunda verticalmente. Isto é claramente visto no painel (B), com o termo de transporte removendo massa de baixos níveis para níveis mais altos. A razão de mistura do traçador diminui ao longo do dia (painel C), na ausência de emissão, tendendo a um perfil vertical homogêneo, característica de uma camada bem misturada.

O transporte de poluentes da CLP para a troposfera livre por nuvens precipitantes ou não, constitui um importante mecanismo que afeta a composição química da troposfera e os

$$
\begin{aligned}
& \left(-\overline{\boldsymbol{u}}_{i} \frac{\partial \overline{\boldsymbol{s}}}{\partial \boldsymbol{x}_{i}}\right)_{\substack{a d v \\
j}} \approx-\frac{1}{\rho_{0} \Delta \boldsymbol{x}_{i}}\left[\left(\left(\rho_{0} \boldsymbol{F}\right)_{j+1 / 2}-\left(\rho_{0} \boldsymbol{F}\right)_{j-1 / 2}\right)-\overline{\boldsymbol{s}}_{j}\left(\left(\rho_{0} \overline{\boldsymbol{u}}_{i}\right)_{j+1 / 2}-\left(\rho_{0} \overline{\boldsymbol{u}}_{i}\right)_{j-1 / 2}\right)\right] \\
& \operatorname{com} \boldsymbol{F}_{j+1 / 2}=\frac{\Delta \boldsymbol{x}_{i}}{\Delta \boldsymbol{t}}\left[\frac{\alpha}{2}\left(\overline{\boldsymbol{s}}_{j}+\overline{\boldsymbol{s}}_{j+1}\right)+\frac{\alpha^{2}}{2}\left(\overline{\boldsymbol{s}}_{j}-\overline{\boldsymbol{s}}_{j+1}\right)\right], \quad \alpha=\overline{\boldsymbol{u}}_{\boldsymbol{i}_{j+1 / 2}} \frac{\Delta t}{\Delta \boldsymbol{x}_{i}}
\end{aligned}
$$



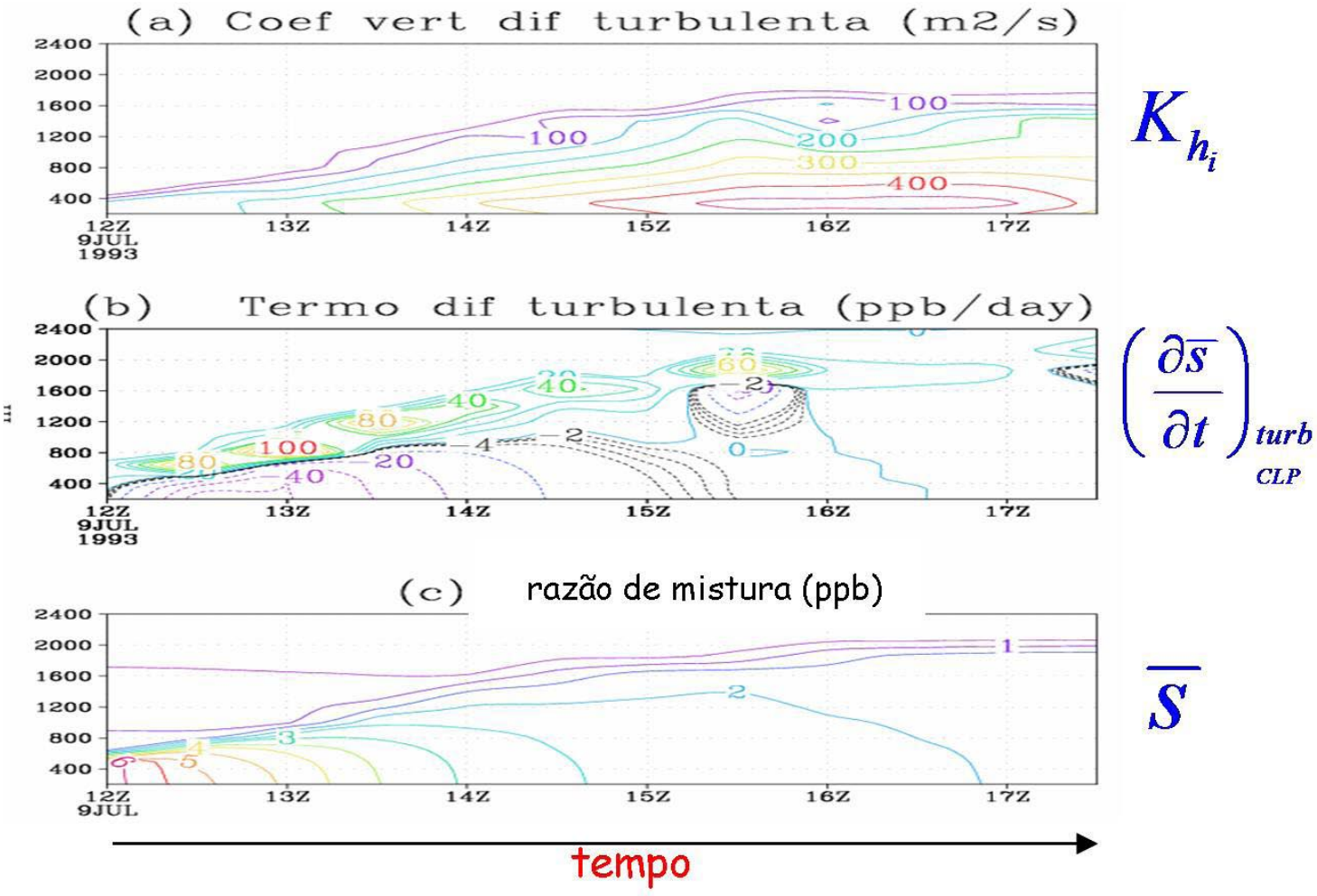

Figura 1 - Ciclo diurno da mistura turbulenta descrita através da simulação numérica do coeficiente de difusividade vertical (A), do termo de transporte turbulento (B) e da razão de mistura do traçador (C).

ciclos biogeoquímicos. A simulação numérica da poluição do ar em escala regional ou global impõe o uso de modelos com baixa resolução horizontal espacial $(\Delta \boldsymbol{x}>10 \mathrm{~km})$ e depende, assim, da parametrização de cumulus para a representação, na escala da grade, de processos convectivos úmidos não resolvidos explicitamente. Desta forma, o transporte e deposição úmida de gases e aerossóis por estes sistemas na escala da sub-grade, precisam também ser parametrizados e incluídos na equação de continuidade. O ponto de partida para a parametrização é o reconhecimento de que, para os processos em questão, apenas o termo vertical é relevante:

$$
\left(\frac{\partial \overline{\boldsymbol{s}}}{\partial \boldsymbol{t}}\right)_{c o n v} \approx-\frac{1}{\rho_{0}} \frac{\partial \rho_{0} \overline{\boldsymbol{w}^{\prime} \boldsymbol{s}^{\prime}}}{\partial \boldsymbol{z}}
$$

e o problema se torna em como estimar os fluxos turbulentos em $\overline{\boldsymbol{w}^{\prime} \boldsymbol{s}}$ função de propriedades da escala da grade. Utilizando o formalismo de fluxo de massa, a decomposição nuvem-ambiente e a aproximação de que os processos convectivos ocupam uma pequena área em relação ao ambiente, é possível escrever a seguinte relação para o fluxo turbulento (Arakawa e Schubert, 1974, Grell, 1993)

$$
\begin{aligned}
& \overline{w^{\prime} s^{\prime}}=m_{c}\left(\bar{s}^{c}-\bar{s}\right) \\
& m_{c}=\rho \sigma \bar{w}^{c}
\end{aligned}
$$

onde $\boldsymbol{m}_{\mathrm{c}}$ é denominado fluxo de massa, $\rho$ é a densidade do ar, $\sigma$ a fração de área ativa dos cumulus, $\overline{\boldsymbol{w}}^{c}$ a velocidade vertical dentro da nuvem, $\overline{\boldsymbol{s}}^{c}$ e $\overline{\boldsymbol{s}}$ as razões de mistura do traçador dentro da nuvem e no ambiente, respectivamente. A generalização deste resultado para incluir todos os tipos de nuvens com correntes ascendentes (updrafts, u) e descendentes (downdrafts, d), leva à seguinte equação (Arakawa e Schubert, 1974)

$$
\begin{aligned}
& \overline{w^{\prime} s^{\prime}}=\int_{\lambda}\left(\overline{s_{u}}-\bar{s}\right) \eta_{u}(\lambda, z) m_{u}\left(\lambda, z_{b, u}\right) d \lambda- \\
& \int_{\lambda}\left(\bar{s}_{d}-\bar{s}\right) \eta_{d}(\lambda, z) m_{d}\left(\lambda, z_{b, d}\right) d \lambda
\end{aligned}
$$

onde $\eta_{u, d}$ corresponde ao fluxo de massa normalizado da corrente ascendente $(\boldsymbol{u}) /$ descendente $(\boldsymbol{d})$ da nuvem e $\boldsymbol{m}_{\boldsymbol{u}, \boldsymbol{d}}$ ao fluxo de massa na base da nuvem (u) ou no nível onde a corrente descendente inicia $(\boldsymbol{d})$. A variável $\lambda$ representa o espectro de nuvens presente na grade do modelo e relevante 
para o problema. Normalmente, as parametrizações de cumulus consideram apenas dois tipos de nuvens no espectro (rasas não-precipitantes e profundas precipitantes), que são tratadas de forma independente. Estabelecendo hipóteses de como o ar ambiente entranha na nuvem e como o ar dentro da nuvem desentranha no ambiente e impondo a conservação de massa no sistema nuvem-ambiente, é possível escrever a seguinte equação para o transporte na sub-grade de traçadores por convecção profunda e rasa, respectivamente:

$$
\begin{aligned}
& \left(\frac{\partial \overline{\boldsymbol{s}}}{\partial \boldsymbol{t}}\right)_{\text {ceep }}=\frac{\boldsymbol{m}_{u, d p}\left(\bar{z}_{b}\right)}{\rho_{0}}[\underbrace{\delta_{u} \eta_{u}\left(\overline{\boldsymbol{s}}_{u}-\overline{\boldsymbol{s}}\right)}_{u p}+\underbrace{\delta_{d} \varepsilon \eta_{d}\left(\overline{\boldsymbol{s}}_{d}-\overline{\boldsymbol{s}}\right)}_{\text {down }}+\underbrace{\tilde{\eta} \frac{\partial \overline{\boldsymbol{s}}}{\partial z}}_{\text {env }}] \\
& \left(\frac{\partial \overline{\boldsymbol{s}}}{\partial \boldsymbol{t}}\right)_{\text {chl }}=\frac{\boldsymbol{m}_{u, s h}\left(\bar{z}_{b}\right)}{\rho_{0}}[\underbrace{\delta_{u} \eta_{u}\left(\overline{\boldsymbol{s}}_{u}-\overline{\boldsymbol{s}}\right)}_{u p}+\underbrace{\tilde{\eta} \frac{\partial \overline{\boldsymbol{s}}}{\partial z}}_{\text {env }}]
\end{aligned}
$$

onde $\delta_{\mathbf{u}, \mathbf{d}}$ expressa a taxa de desentranhamento de massa da nuvem no ambiente das correntes ascendentes $(\boldsymbol{u})$ e descendentes (d), e o termo denotado por env representa o transporte do traçador no ambiente pela subsidência compensatória deste. O modelo de nuvens adotado pela parametrização determina a estrutura vertical do transporte. Contudo, a magnitude do transporte é determinada pelo fluxo de massa na base de nuvens $\left(\boldsymbol{m}_{u, d p / s h}\right)$, o qual é, por sua vez, obtido pela hipótese de fechamento (por exemplo, quase-equilíbrio) assumida pela parametrização convectiva. Note que o transporte por correntes descendentes, não é normalmente considerado no caso de cumulus rasos não precipitantes. Para ilustrar a simulação do transporte por convecção profunda, a Figura $2 \mathrm{~A}$ apresenta um perfil vertical de razão de mistura de CO desde a CLP até a alta troposfera. Trata-se de uma simulação real para as 2100 UTC do dia $24 / 09 / 2002$, e o perfil foi tomado ao longo da latitude $10 \mathrm{~S}$. O CO simulado é decorrente de emissões de queimadas bastante freqüentes nesta época do ano sobre a região Norte do Brasil, e se concentra entre as longitudes $70 \mathrm{~W}$ e $50 \mathrm{~W}$. Dentro dos primeiros $2 \mathrm{~km}$ de altura, $\mathrm{CO}$ é relativamente bem misturado com concentrações variando entre 500 e 1000 ppb, dependendo da proximidade de fontes. $\mathrm{Na}$ alta troposfera, como resultado do transporte convectivo, há um enriquecimento de $\mathrm{CO}$ da ordem 4 a 6 vezes o valor do ambiente. A camada enriquecida, neste caso, situa-se entre $9 \mathrm{~km}$ e $13 \mathrm{~km}$ de altura, região típica de desentranhamento de sistemas profundos. Desta forma, emissões de gases do efeito estufa e outros poluentes tóxicos, são injetados na alta troposfera e transportados rapidamente para longas distâncias das fontes pelos ventos intensos presentes. Este transporte é ilustrado na Figura 2B, onde aparece a pluma de $\mathrm{CO}$ em 11,5 km e as linhas de corrente da circulação sobre a América do Sul. A Figura 3 apresenta resultados similares para a convecção rasa. No painel (A) é mostrada a simulação numérica do transporte de $\mathrm{CO}$, proveniente de queimadas da CLP para a baixa troposfera por sistemas convectivos rasos. No painel (B)
(A) Monóxido de Carbono (ppbv) Lat 10S - 21 UTC 24/09/2002

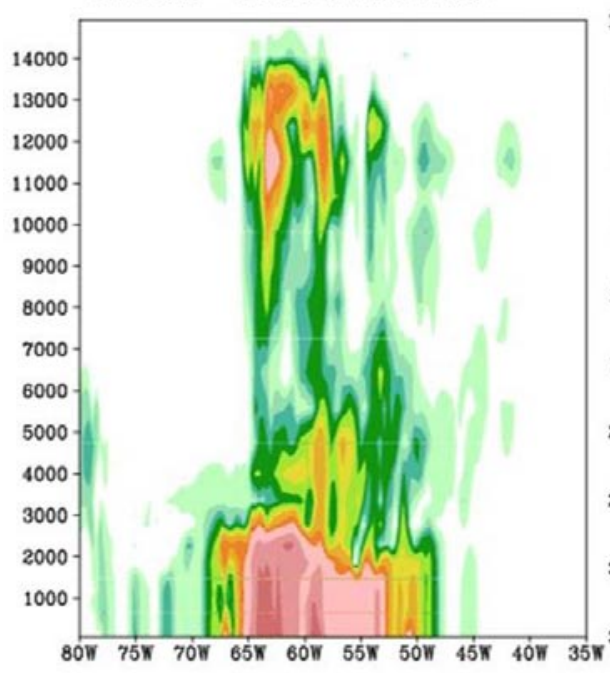

(B) Monóxido de Carbono (ppbv) Alt. 11,5 km - 21 UTC 24/09/2002

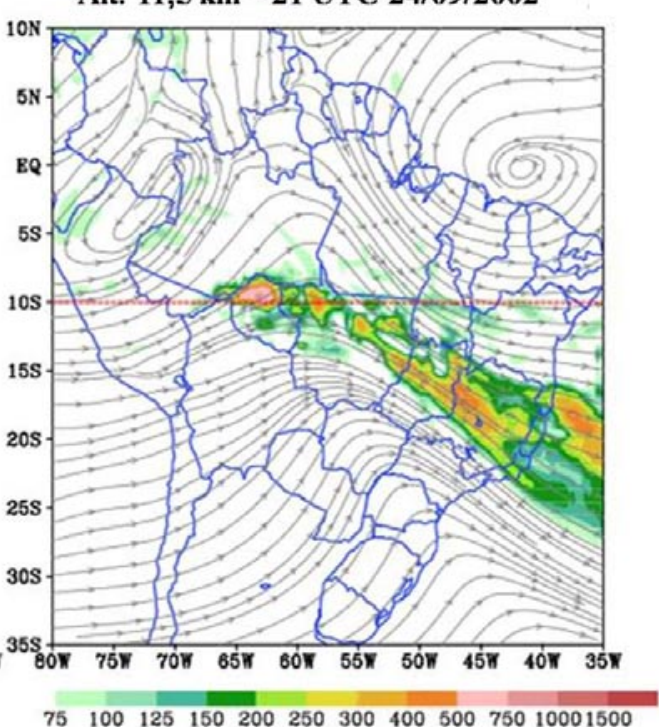

Figura 2 - (A) Exemplo do transporte vertical de gases traço por convecção profunda. (B) transporte horizontal em 11.5 km de CO introduzido neste nível por sistemas convectivos em Rondônia. 
apresenta-se uma combinação de dois campos: as isolinhas em preto são os fluxos de massa na base das nuvens rasas utilizado aqui para demarcar a ocorrência espacial destas; sombreado em cores denota a razão de mistura de CO ao nível de $3300 \mathrm{~m}$ acima da superfície, altura típica da região de desentranhamento de massa transportada por estes sistemas convectivos. O painel (A) apresenta o perfil vertical ao longo da longitude $65 \mathrm{~W}$ simulado para as 1800 UTC do dia 03/09/2003. Entre as latitudes $17 \mathrm{~S}$ e $14 \mathrm{~S}$, o transporte vertical decorre somente da turbulência na CLP. Ao norte de $14 \mathrm{~S}$, cumulus rasos são simulados e atuam sobre a CLP poluída transportando CO para a baixa troposfera, conforme mostrado neste painel. Observa-se também no painel (B), CO sendo transportado para o Sul pela circulação regional presente na baixa troposfera.

\subsection{Descrição dos termos forçantes}

O termo $\overline{\boldsymbol{Q}_{s}}$ representa processos físicos e/ou químicos não descritos em termos de transporte tais como emissão, deposição seca e úmida, reatividade química e de microfísica de aerossóis que, no entanto, modificam localmente a razão de mistura do traçador.

Importantes processos de emissão de poluentes para a atmosfera incluem queimadas (naturais ou antrópicas), outras formas de combustão (transporte, industrial, produção de carvão, etc.) e processos biogênicos. Em queimadas, a principal emissão acontece na forma de $\mathrm{CO}_{2}$, produzido principalmente na fase de chamas. As emissões deste composto representam em média cerca de $80 \%$ a $85 \%$ da massa total de carbono queimado, podendo, no entanto, variar de $50 \%$ a $99 \%$. A emissão de carbono na forma de $\mathrm{CO}$ representa em média $7 \%$, podendo variar entre $2 \%$ a $15 \%$. Em terceiro lugar, aparecem os hidrocarbonetos com médias em torno de $2 \%$ a $3 \%$, com $\mathrm{CH}_{4}$ representando cerca de $0,5 \%$. Outros gases emitidos incluem o $\mathrm{N}_{2} \mathrm{O}$ e $\mathrm{NO}_{\mathrm{x}}$ e também material particulado. Uma característica relevante das emissões de queimadas é o forte empuxo inicial associado às altas temperaturas dos gases emitidos. Isto impõe uma forte aceleração inicial, que injeta parte da emissão diretamente na CLP, troposfera livre ou mesmo na estratosfera (FROMM et al., 2000). A determinação da altura final de injeção constitui uma informação crucial e depende, principalmente, da estabilidade termodinâmica da atmosfera, do vento horizontal e da quantidade de energia térmica liberada pela combustão. Chatfield and Delany (1990) e Poppe et al. (1998) demonstraram que devido à não-linearidade da produção de ozônio, sua taxa de formação é influenciada pela diluição e transporte atmosféricos. Assim, uma descrição correta da altura de injeção de emissões quentes é necessária para a simulação da reatividade química e transporte subseqüente. Na ausência deste mecanismo, as emissões pirogênicas são freqüentemente liberadas na superfície do modelo, ou verticalmente distribuídas em uma forma arbitrária (Turquety et al., 2007), ou usando alguma relação empírica entre a altura de injeção e a intensidade do fogo (Lavoué et al., 2000). Freitas et al. (2007) apresentaram uma metodologia baseada no uso de um modelo de nuvens 1D embebido no modelo de transporte 3D. Nesta técnica, é possível estimar a camada de injeção partindo das informações de fluxo de calor, tamanho do fogo e do ambiente onde este está ocorrendo. Após cessarem as chamas, uma fase mais fria, com brasas e de menor eficiência de combustão, persiste, emitindo gases e aerossóis que são liberados proximamente à superfície e são misturados dentro da CLP pela turbulência.

Emissões urbanas, industriais, veiculares e biogênicas são normalmente mais frias, sendo lançadas próximas à superfície
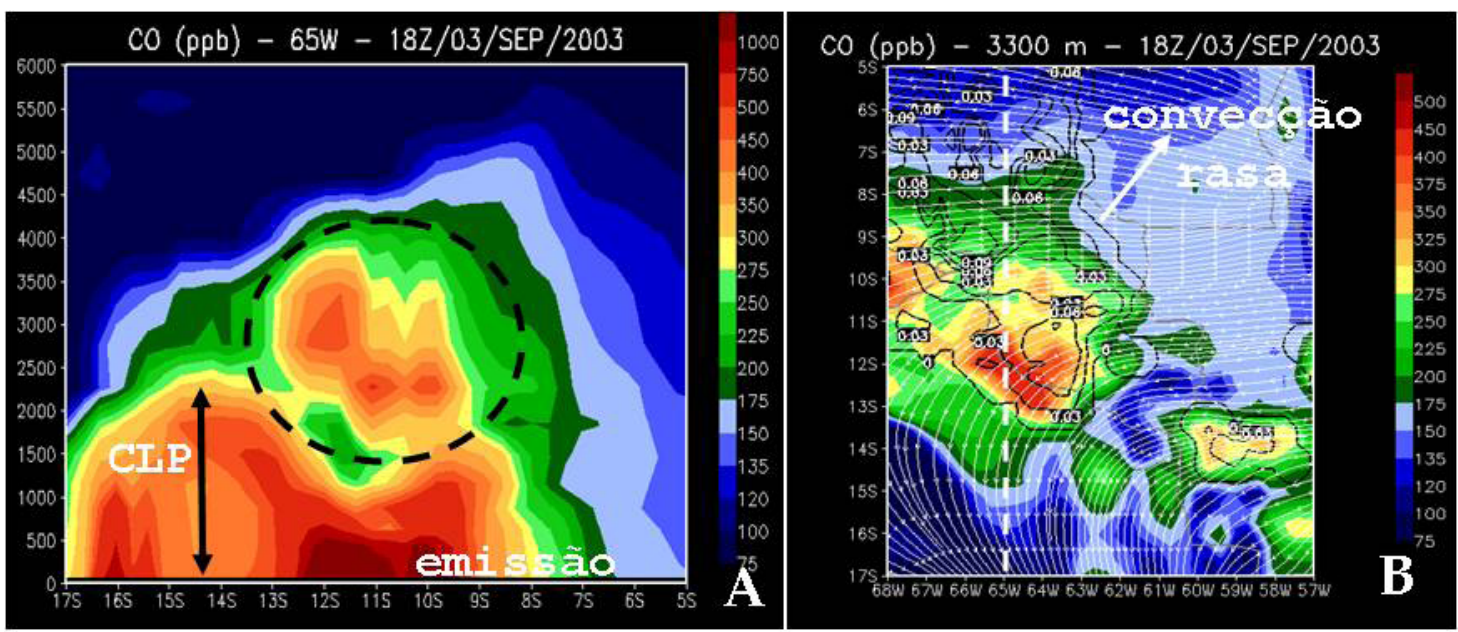

Figura 3 - (A) Simulação numérica do transporte de CO da camada limite planetária para a baixa troposfera por sistemas convectivos rasos, descritos pelas isolinhas de fluxos de massa (em preto) na figura (B). 
dentro da CLP transportadas verticalmente pela turbulência presente. Existem diversos inventários destas emissões em várias escalas espaciais e temporais.

O programa "REanalysis of the TROpospheric chemical composition" (RETRO, retro.enes.org), constitui um excelente exemplo do esforço internacional para a geração de inventários de emissão de diversas espécies químicas relevantes para a atmosfera e qualidade do ar. Trata-se de um inventário global (resolução de 0,5 grau) com totais mensais de massa de $\mathrm{CO}_{2}$, $\mathrm{CO}, \mathrm{NO}_{\mathrm{X}}, \mathrm{CH}_{4}$, e compostos orgânicos voláteis emitida por ponto de grade. Estimativas de emissões biogênicas podem ser obtidas a partir do programa Global Emissions Inventory Activities (GEIA, www.geiacenter.org), embora emissões interativas, on-line, com modelo atmosférico, produzidas por sistemas como o Model of Emissions of Gases and Aerosols from Nature (MEGAN, Guenther et al., 1999) possam ser mais representativas para uso em modelos regionais e na escala de tempo.

Para emissões com empuxo desprezível em relação ao ambiente, o termo de emissão $\boldsymbol{E}_{\boldsymbol{\eta}}$ de uma espécie química $\boldsymbol{\eta}$ pode ser definido como uma emissão na superfície da seguinte forma:

$$
\boldsymbol{E}_{\eta}= \begin{cases}\frac{\boldsymbol{F}_{\eta}}{\rho_{0} \Delta \boldsymbol{z}_{1}} r(\boldsymbol{t}), & \boldsymbol{k}=1 \\ 0, & \boldsymbol{k}>1\end{cases}
$$

onde $\boldsymbol{F}_{\boldsymbol{\eta}}$ representa o fluxo de massa $\left(\mathrm{em} \mathrm{kg}[\boldsymbol{\eta}] \mathrm{m}^{-2}\right)$ total emitido diariamente dentro do volume de grade, $\Delta z_{1}$ a espessura vertical da primeira célula de grade do modelo e $\mathbf{r}(\mathbf{t})$ uma função normalizada a 1, que representa o ciclo diurno da emissão. Normalmente, esta função é descrita como uma Gaussiana dupla para as emissões urbanas, com picos no início da manhã e fim de tarde, representando os horários de trânsito mais intenso de veículos automotores nas cidades. Emissões por aeronaves requerem tratamento diferenciado para a emissão vertical, sendo a altitude de cruzeiro uma informação necessária para definição correta da camada de injeção dos poluentes emitidos. Queimadas também necessitam de tratamento diferenciado, pois os traçadores emitidos durante a fase das chamas podem ter empuxo inicial suficiente para serem transportados diretamente acima da superfície, conforme descrito anteriormente. Neste caso, primeiramente a fração de massa emitida na fase das chamas $(\lambda)$ é estimada. Com o uso de um modelo de levantamento de plumas, a altura $h$ da injeção e a espessura da camada de injeção $\left(\Delta z_{h}\right)$ são estimadas (veja Figura 4 para uma ilustração do processo), sendo possível definir o termo de emissão com dependência da coordenada vertical do modelo (Equação 13).

$$
\boldsymbol{E}_{\eta}=\left\{\begin{array}{lr}
\frac{(1-\lambda) F_{\eta}}{\rho_{0} \Delta z_{1}} r(t) & , \boldsymbol{k}=1 \\
\frac{\lambda \boldsymbol{F}_{\eta}}{\rho_{0} \Delta z_{\mathrm{h}}} r(t) & , \boldsymbol{h}-\frac{\Delta z_{\mathrm{h}}}{2}<z(\boldsymbol{k})<\boldsymbol{h}+\frac{\Delta \boldsymbol{z}_{h}}{2}
\end{array}\right.
$$

Neste caso, a função $\mathbf{r}(\mathbf{t})$ representa o ciclo diurno da emissão por queimadas sendo representado por uma Gaussiana simples com máximo em torno do meio da tarde, horário de pico da ocorrência de focos de queimadas na América do Sul (Prins et al., 1998). A estimativa do fluxo de emissão $\boldsymbol{F}_{\boldsymbol{\eta}}$ associado às queimadas pode ser obtida a partir da relação (Freitas et al., 2005, Longo et al., 2007)

$$
F_{\eta}=\frac{\sum_{i} \alpha_{v e g_{i}} \cdot \beta_{v g_{i}} \cdot \mathrm{E}_{f_{v g_{i}}}^{\eta} \cdot a_{i}}{\Delta x \Delta y}, i \in \Delta x \Delta y
$$

onde $\alpha_{v e g i}$ é a quantidade de biomassa seca acima do solo na posição do fogo i, $\beta_{v e g i}$ a fração de biomassa efetivamente consumida, $\mathrm{E}_{v_{v g_{i}}}^{\eta} \mathrm{o}$ fator de emissão que expressa a quantidade de massa do gás $\eta$ emitido por unidade de biomassa seca consumida e $\boldsymbol{a}_{i}$ a área queimada pelo fogo $\mathbf{i}$. O termo $\Delta x \Delta y$ representa a área da célula do modelo e a soma é efetuada sobre todos os focos de queimada que estão dentro de cada célula. Para áreas de floresta tropical, números típicos para $\alpha_{v e g i}$ e $\beta_{v e g i}$ são 30 e $0.5 \mathrm{~kg} \mathrm{~m}^{-2}$, respectivamente. Como exemplo, para uma queimada de 10 ha, a quantidade de $\mathrm{CO}$ emitida, utilizando um fator de emissão da ordem de $100 \mathrm{~g} \mathrm{~kg}^{-1}$, é em torno de 150 ton, o que é suficiente para elevar em 2 - 3 ordens de grandeza a razão de mistura localmente. A estimativa dada pela Equação (14) apresenta um significativo grau de incerteza associada, principalmente, à área queimada. Fatores de emissão para diversos tipos de bioma são descritos em vários trabalhos (por exemplo, Andreae e Merlet, 2001), porém, similarmente ao fator combustão, apresentam uma significativa dependência com o grau de umidade presente na vegetação. Quanto maior o conteúdo de água na biomassa,

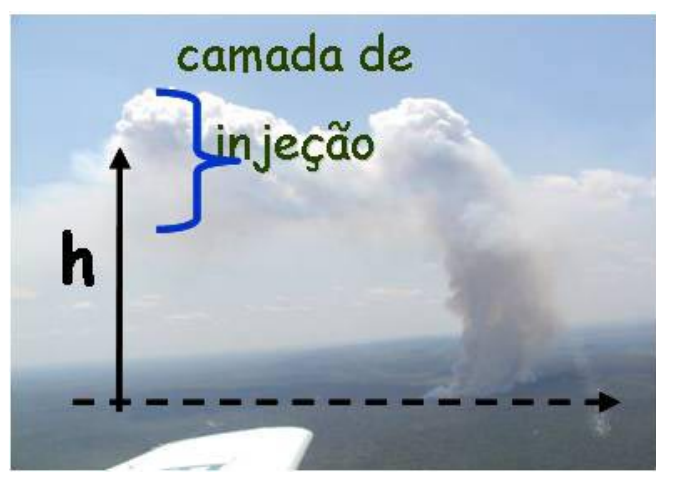

Figura 4 - Ilustração do processo de plume rise de emissões de queimadas com definição da altura e camada de injeção. 
mais energia é alocada no processo de evaporação desta, reduzindo a temperatura da combustão e aumentado a proporção de compostos incompletamente oxidados emitidos.

O estado-da-arte da estimativa do conteúdo de biomassa em regiões de floresta tropical e cerrado da América do Sul, é dado em Saatchi et al. (2007). Neste estudo, os autores utilizaram dados de sensoriamento remoto validados com medidas de campos e geraram uma base de dados de densidade de biomassa $\left(\mathrm{kg} \mathrm{m}^{-2}\right)$ com $1 \mathrm{~km}$ de resolução espacial.

A Figura 5 apresenta estimativas de emissão de monóxido de carbono de origem antrópica (urbano, industrial e transporte), por queimadas e por processos naturais biogênicos para setembro de 2002. Dados em resolução espacial de 50 x 50 $\mathrm{km}^{2}$, em umidades $10^{-6} \mathrm{~kg}[\mathrm{CO}] \mathrm{m}^{-2}$ e, no caso de queimadas, utilizou-se focos obtidos por sensoriamento remoto para o dia 21 do referido mês. A estimativa de emissões, utilizando a energia radiativa do fogo obtida por sensores a bordo de satélites (Ichoku e Kauffamnn, 2005, Pereira, 2008), constitui uma técnica recente e promissora, embora também sujeita a grandes incertezas, principalmente na taxa de conversão de energia radiativa para fluxo de material particulado ou de $\mathrm{CO}_{2}$.

A atmosfera é composta de diversos gases que possuem a propriedade de reagirem por processos cinéticos e/ou por fotodissociação, formando espécies secundárias. Em processos cinéticos, a colisão entre moléculas é uma condição necessária para a reação. Além disto, estas devem ter energia suficiente para quebrar as ligações químicas. A fotodissociação é o processo no qual a interação de fótons com moléculas pode levá-las a um estado excitado que, na seqüência, as dissocia, dando origem a outras espécies. Porém, a dissociação não constitui o único processo possível, pois a molécula pode simplesmente re-emitir a energia do fóton e voltar para o estado fundamental ou pode colidir com o meio e liberar a energia extra recebida. A ionização da molécula, também é outro processo possível de ocorrer numa reação fotoquímica.

As taxas de reação química determinam se uma espécie é formada ou destruída nos processos de reação, e as reações são normalmente classificadas em ordens em função do número de moléculas envolvidas. Um exemplo de reação de primeira ordem (uni-molecular) seria a reação de fotólise:

$$
A+\boldsymbol{w} \rightarrow B
$$

onde a molécula A, ao absorver um fóton (de energia $\boldsymbol{h}$ ) se dissocia, dando origem à molécula $\mathrm{B}$. Matematicamente, a expressão para o balanço de massa é dada pelo sistema de equações diferenciais

$$
\begin{aligned}
& \frac{d \rho_{A}}{d t}=-j \rho_{A} \Rightarrow \text { destruição de } A \\
& \frac{d \rho_{B}}{d t}=+j \rho_{A} \Rightarrow \text { produção de } B
\end{aligned}
$$

onde $\rho$ é a concentração das espécies, expressa normalmente em molec $\mathbf{c m}^{-3}$, e $\boldsymbol{j}$ a taxa de fotólise $\left(\boldsymbol{s}^{-1}\right)$. As taxas de fotólise, quantitativamente definidas como as freqüências de reações de fotólise, são obtidas através do uso da equação de transferência radiativa.

As taxas de reação cinéticas são expressas numa forma que inclui a freqüência de colisão entre moléculas e a fração

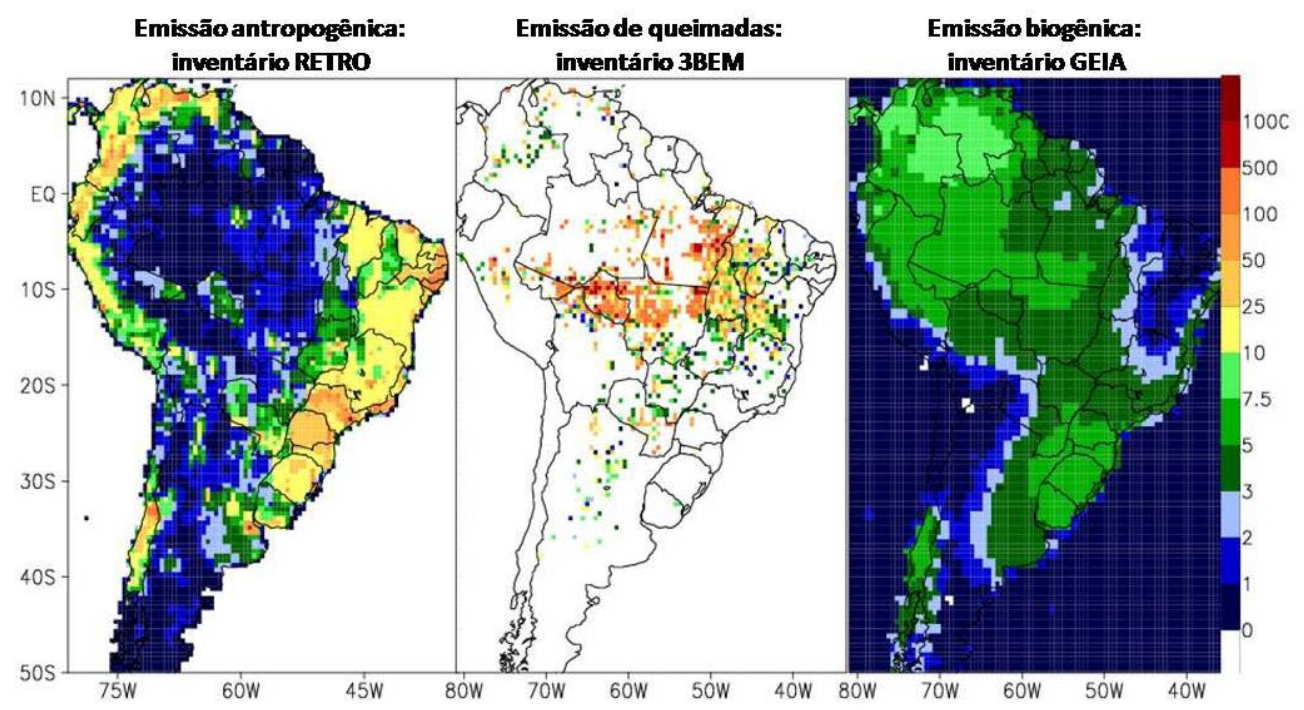

Figura 5 - Estimativas de emissão de monóxido de carbono de origem antrópica (urbano, industrial e transporte), por queimadas e por processos naturais biogênicos para setembro de 2002 . Dados em $50 \times 50 \mathrm{~km}^{2}$, em $10^{-6} \mathrm{~kg} \mathrm{~m}^{-2}$ e no caso de queimadas utilizou-se focos obtidos por sensoriamento remoto para o dia 21 . 
destas, que excedem a energia requerida para a quebra de ligações químicas. Assim, são normalmente dependentes da temperatura e da pressão atmosféricas. Um exemplo de reação de segunda ordem é dado abaixo, onde as moléculas A e B reagem cineticamente, dando origem às moléculas $\mathbf{C}$ e $\mathbf{D}$

$$
A+B \rightarrow C+D
$$

com taxa de reação dada por $\boldsymbol{k}_{\boldsymbol{s}} \rho_{\boldsymbol{a}} \rho_{\boldsymbol{b}} \boldsymbol{e} \boldsymbol{k}_{\boldsymbol{s}}=\boldsymbol{f}(\boldsymbol{T}, \boldsymbol{p})$, sendo o coeficiente de reação dependente da temperatura $(\boldsymbol{T})$ e pressão (p). Neste exemplo, o balanço de massa é dado por:

$$
\begin{aligned}
& \frac{d \rho_{A}}{d t}=\frac{d \rho_{B}}{d t}=-k_{s} \rho_{A} \rho_{B}, \Rightarrow \text { destruição de A e B } \\
& \frac{d \rho_{C}}{d t}=\frac{d \rho_{D}}{d t}=+k_{s} \rho_{A} \rho_{B}, \Rightarrow \text { produção de C e D }
\end{aligned}
$$

A formação do ozônio $\left(\mathrm{O}_{3}\right)$ na troposfera constitui um importante exemplo da reatividade química com impacto na poluição do ar. O ozônio é altamente tóxico, implicando em efeitos na saúde humana quando respirado e também produz degradação de plantas presentes em regiões contaminadas. Em termos de balanço radiativo, ele absorve radiação ultravioleta nociva a seres vivos e absorve também no infra-vermelho, sendo portanto um dos gases do efeito estufa. No relatório do IPCC de 2007, o $\mathrm{O}_{3}$ é o terceiro componente da forçante radiativa com valor médio estimado em $+0,35 \mathrm{~W} \mathrm{~m}^{-2}$. Em termos gerais, as condições propícias para formação de $\mathrm{O}_{3}$ requerem presença de óxidos nitrosos $\left(\mathrm{NO}_{\mathrm{x}}=\mathrm{NO}+\mathrm{NO}_{2}\right)$, compostos orgânicos voláteis (COV) e radiação solar ( $\lambda<420 \mathrm{~nm}$ ). A interação destes três componentes inicia uma complexa cadeia de reações que dá origem à formação de $\mathrm{O}_{3}$. $\mathrm{O}$ sistema de reações químicas (16) apresenta uma simplificação deste processo. $\mathrm{NO}_{x}$ são tipicamente emitidos em processos de combustão (queimadas, motores automotivos, etc), enquanto que os $\mathrm{COV}$ são emitidos, como exemplo, por evaporação de solventes e atividades biogênicas. Em geral, na ausência de COV, não há uma produção líquida de $\mathrm{O}_{3}$ com somente $\mathrm{NO}_{\mathrm{X}}$ e radiação solar, isto é, considerando somente as reações 3, 4 e 5. No entanto, na presença de $\mathrm{COV}$ inicia-se uma série de reações extras que terminam numa produção líquida de $\mathrm{NO}_{2}$ e consumo de NO (reação 2) implicando em um desbalanço que favorece a formação de $\mathrm{O}_{3}$.

$$
\left.\begin{array}{l}
\text { (1) } \mathbf{C O V} \stackrel{\text { fotólise }}{\text { cinética }} \mathbf{C O V}^{*} \text { (radicais livres) } \\
\text { (2) } \mathrm{COV}^{*}+\mathrm{NO} \longrightarrow \mathrm{NO}_{2}+\mathbf{C O V}^{* *} \\
\text { (3) } \mathrm{NO}+\mathrm{O}_{3} \longrightarrow \mathrm{NO}_{2}+\mathrm{O}_{2} \\
\text { (4) } \mathrm{NO}_{2}+m \longrightarrow \mathrm{NO}+\mathbf{O}(\lambda<420 \mathrm{~nm}) \\
\text { (5) } \left.\mathbf{O}+\mathrm{O}_{2}+\mathrm{M} \longrightarrow \mathrm{O}_{3}+\mathrm{M} \text { (formação líquida de } \mathrm{O}_{3}\right)
\end{array}\right\}
$$

A inclusão da reatividade química constitui uma das tarefas mais difíceis em modelagem numérica da poluição do ar. $\mathrm{O}$ termo de reatividade química é expresso pelo seguinte sistema de equações diferenciais não-lineares acopladas:

$$
\begin{aligned}
& \left(\frac{\partial \rho_{k}}{\partial t}\right)_{\text {chem }} \equiv \frac{d \rho_{k}}{d t}=P_{k}(\vec{\rho})-L_{k}(\vec{\rho}), \quad k=1, N \\
& \vec{\rho}=\left\{\rho_{1}, \rho_{2}, \ldots, \rho_{N}\right\} \\
& \text { com a condição inicial: } \\
& \vec{\rho}\left(t_{0}\right)=\left\{\rho_{1}\left(t_{0}\right), \rho_{2}\left(t_{0}\right), \ldots, \rho_{N}\left(t_{0}\right)\right\}
\end{aligned}
$$

onde $\rho_{\boldsymbol{k}}$ representa a densidade número da espécie $\boldsymbol{k}$ (molec/ $\mathbf{c m}^{3}$ ), $\mathrm{N}$ representa o número de espécies químicas reagentes, $\boldsymbol{P}_{\boldsymbol{k}}$ corresponde à produção líquida da espécie $\boldsymbol{k}$ e $\boldsymbol{L}_{\boldsymbol{k}}$ ao termo líquido de perda de $\boldsymbol{k}$. Pelo fato das taxas de reações químicas, ocorrendo na atmosfera variarem em diversas ordens de grandezas, o sistema de Equações 17 é descrito como um sistema stiff(rígido) exigindo métodos implícitos para solução numérica não proibitiva. Além disto, mecanismos químicos completos para a fase gasosa e adequados para a troposfera em escala regional, como, por exemplo, o Regional Atmospheric Chemistry Mechanism (RACM, Stockwell et al., 1997), possuem da ordem de 70 espécies reagindo através de 230 reações cinéticas ou por fotólise. Isto implica no alto custo computacional envolvido na solução de (Equações 17), sendo de longe o processo mais dispendioso, em termos computacionais, na solução da equação de continuidade (Equação 3). Dentro das diversas técnicas de solução numérica implícita de (Equações 17), se destaca o método de Rosenbrock (Hairer e Wanner, 1991). O método de Rosenbrock é um método implícito de múltiplos estágios que permite a troca da solução de um sistema de equações diferenciais não lineares (Equações 17) por um sistema linear algébrico em termos de incrementos $\boldsymbol{K}_{\boldsymbol{i}}$, sendo a solução dada por:

$$
\vec{\rho}\left(t_{0}+\tau\right)=\vec{\rho}\left(t_{0}\right)+\sum_{i=1}^{s} b_{i} \mathbf{K}_{i}
$$

onde $\boldsymbol{s}$ é o número total de estágios, $\boldsymbol{b}_{\boldsymbol{i}}$ são constantes numéricas dependentes de $\boldsymbol{s}$ e $\tau$ o passo de integração no tempo. Os incrementos $\boldsymbol{K}_{\boldsymbol{i}}$ são obtidos seqüencialmente através da solução do sistema linear algébrico dado por:

$$
\left\{\begin{array}{l}
\mathbf{K}_{i}=\tau \mathbf{F}\left(\vec{\rho}_{i}\right)+\tau \mathbf{J}\left(\vec{\rho}\left(t_{0}\right)\right) \cdot \sum_{j=1}^{i} \gamma_{i j} \mathbf{K}_{j} \\
\vec{\rho}_{i}=\vec{\rho}\left(t_{0}\right)+\sum_{j=1}^{i-1} \alpha_{i j} \mathbf{K}_{j} \\
\mathbf{F}\left(\vec{\rho}_{i}\right)=\vec{P}\left(\vec{\rho}_{i}\right)-\vec{L}\left(\vec{\rho}_{i}\right)
\end{array}\right.
$$


onde $\boldsymbol{i}=\mathbf{1}, \boldsymbol{s}, \alpha_{i j} \boldsymbol{e} \boldsymbol{y}_{\boldsymbol{i} \boldsymbol{j}}$ são constantes dependentes do número de estágios $s, \vec{\rho}_{i}$ é uma solução intermediária utilizada para recalcular a produção líquida no estágio $\boldsymbol{i}$ dada pelo termo $\mathbf{F}\left(\vec{\rho}_{i}\right)$ e $\boldsymbol{J}$ a matriz Jacobiano da produção líquida no tempo t0. A maior parte do esforço computacional envolve a solução do sistema linear descrito na primeira equação de (19). O sistema linear a ser resolvido é da forma:

$$
A \boldsymbol{x}=\boldsymbol{b}
$$

onde $\boldsymbol{A}$ é uma matriz N x N, $\boldsymbol{x}$ é o vetor solução e $\boldsymbol{b}$ é o vetor de termos independentes. Porém, duas características relevantes deste sistema podem ser utilizadas para acelerar o processamento. Uma delas é a que o Jacobiano é extremamente esparso, tipicamente $10 \%$ dos elementos são não nulos. A segunda é a de que a estrutura da matriz é invariável no tempo e espaço. Isto permite o conhecimento e mapeamento prévio dos elementos não nulos sobre os quais deverão ser realizadas as operações matriciais. Para a solução do sistema de Equações 20 pode, por exemplo, utilizar o método desenvolvido por Kundert e Sangiovanni-Vincentelli (1986, 1988). Neste método, tomando como referência as posições fixas onde ocorrem as reações químicas, se perfaz uma fatoração LU e resolve o sistema de matrizes trabalhando com ponteiros de memória. $\mathrm{O}$ método trabalha sobre três matrizes originárias da matriz a ser resolvida: uma matriz diagonal, uma triangular inferior e outra triangular superior:

$$
\boldsymbol{A}=\boldsymbol{D}+\boldsymbol{L}+\boldsymbol{U}
$$

com a solução dada por:

$$
\boldsymbol{x}^{(k+1)}=\boldsymbol{D}^{-1}\left[-(\boldsymbol{L}+\boldsymbol{U}) \boldsymbol{x}^{(k)}+\boldsymbol{b}\right] \quad, \boldsymbol{k}=1,2, . .
$$

Os elementos da matriz são alocados de forma dinâmica uma única vez e eliminam-se as iterações desnecessárias. Um índice de ponteiros é previamente criado a fim de relacionar a seqüência das posições alocadas, na memória com sua posição original na matriz. A ordem de fatoração das linhas e colunas de uma matriz é de extrema importância afetando diretamente o tempo e a precisão do resultado. $\mathrm{O}$ método resolve a matriz em duas partes, decomposição (ou fatoração) e o integrador utiliza o método de substituição forward-backward.

A deposição seca e a úmida constituem importantes sumidouros de gases e particulados da atmosfera. Deposição seca de gases e partículas constitui no transporte destes materiais da atmosfera para superfícies, sendo os principais fatores controlando este processo a turbulência atmosférica, as propriedades químicas das espécies e a natureza da superfície receptora. O fluxo de deposição $\boldsymbol{f}_{\boldsymbol{d} d \boldsymbol{d}}$ de material para superfície é geralmente descrito pela Equação 23

$$
f_{d d}=-v_{d} \bar{s}_{z_{r e f}},
$$

onde $\boldsymbol{v}_{\boldsymbol{d}}$ é denominada velocidade de deposição $(\mathrm{m} / \mathrm{s})$ e $\overline{\boldsymbol{s}}_{z_{\text {ref }}}$ a razão de mistura da espécie em alguma altura de referência (tipicamente 2 metros acima da superfície). Uma vez estimado o fluxo de deposição, o termo sumidouro correspondente na Equação 3, pode ser calculado por:

$$
\boldsymbol{R}_{d \boldsymbol{d}}=\left(\frac{\partial \overline{\boldsymbol{s}}}{\partial \boldsymbol{t}}\right)_{\substack{\text { dep. } \\ \text { seca }}}=\frac{\boldsymbol{f}_{d \boldsymbol{d}}}{\Delta \boldsymbol{z}_{1}}
$$

onde $\Delta \boldsymbol{z}_{1}$ se refere à espessura da primeira camada vertical do modelo. Parametrizar o processo de deposição seca em termos da velocidade de deposição constitui a tarefa difícil neste tema, uma vez que toda uma série de complexos processos físicos e químicos é expressa em apenas um termo. O termo $\boldsymbol{v}_{\boldsymbol{d}}$ para deposição gasosa é, normalmente, expresso na forma de um conjunto em série de resistências (análogo a circuitos elétricos), que representam os diversos mecanismos de transporte $\mathrm{e}$ processos de superfície envolvidos:

$$
v_{d}=\frac{1}{R_{a}+R_{b}+R_{c}}
$$

onde os termos $\boldsymbol{R}_{\boldsymbol{a}} \boldsymbol{R}_{\boldsymbol{b}}$ e $\boldsymbol{R}_{\boldsymbol{c}}$ constituem a resistência aerodinâmica dentro da camada superficial da CLP, a resistência à difusão molecular dentro da camada quase-laminar adjacente à superfície e a resistência superficial associada ao processo de absorção ou adsorção pela superfície, respectivamente. A resistência aerodinâmica é determinada, principalmente, pela turbulência que transporta material da atmosfera em direção à superfície. Desta forma, esta é determinada em termos da estabilidade atmosférica próxima à superfície e da rugosidade desta, em função de propriedades termodinâmicas e do vento através de teoria de similaridade. Dentro da fina camada quase-laminar adjacente à superfície, o transporte de gases ocorre por difusão molecular e, desta forma, a resistência depende da difusividade molecular do gás. A resistência superficial é a mais complexa de ser estimada sendo fortemente dependente das características da superfície e químicas da espécie sendo depositada. Valores típicos da velocidade de deposição para o O3, por exemplo, são 0.4 e $0.07 \mathrm{~cm} / \mathrm{s}$ sobre continente e oceano, respectivamente.

Devido à ação gravitacional, partículas suficientemente pesadas não se movem verticalmente com a mesma velocidade das massas de ar, ao contrário dos gases. Estas rapidamente entram num regime de equilíbrio entre as forças gravitacional e de arraste associada à resistência do ar, com uma velocidade terminal dada por, considerando a atmosfera em repouso,

$$
v_{\text {sed }}=\frac{2 r^{2}\left(\rho_{p}-\rho_{a r}\right) g}{9 \eta_{a r}} G_{i}
$$


onde $\boldsymbol{r}$ é o raio da partícula, $\rho_{p}, \rho_{a r}$ constituem a densidade da partícula e do ar, respectivamente, $\boldsymbol{g}$ é aceleração gravitacional, $\eta_{a r}$ é a viscosidade dinâmica do ar (dependente da temperatura) e $\boldsymbol{G}_{\boldsymbol{i}}$ é um fator para corrigir desvios do regime de Stokes, no qual o raio da partícula é muito maior que o livre caminho médio do ar. A Equação 26 é uma aproximação válida para regimes de baixo número de Reynolds ( $<10^{-2}$, Pruppacher e Klett, 1997). No caso de partículas com velocidade de sedimentação não desprezível, o termo de advecção vertical utiliza uma velocidade vertical efetiva dada por

$$
w_{e f}=w_{a r}-v_{s e d}
$$

onde $\boldsymbol{w}_{\boldsymbol{a}}$ representa a velocidade vertical das massas de ar. Sedimentação gravitacional constitui um caminho adicional, paralelo, para o processo de deposição seca de partículas e, neste caso, a velocidade de deposição é, normalmente, dada por

$$
v_{d d, p a r t}=\frac{1}{R_{a}+R_{b}+R_{a} R_{b} v_{s e d}}+v_{s e d}
$$

onde todos os termos já foram definidos previamente.

$\mathrm{O}$ processo de deposição úmida acontece quando o material em questão está embebido dentro de uma porção de água condensada em precipitação; removendo-se, assim, este material da atmosfera e depositando-o sobre a superfície abaixo. Para gases, assume-se um estado de equilíbrio entre as fases gasosa e aquosa dada pela lei de Henry:

$$
\rho_{\text {fase }}^{\text {aquosa }}=\boldsymbol{k}_{\boldsymbol{H}} \boldsymbol{r}_{\text {lw }} \rho_{\substack{\text { fase } \\ \text { gassa }}}
$$

onde $\boldsymbol{k}_{\boldsymbol{H}}$ é a solubilidade da espécie a uma dada temperatura, e $\boldsymbol{r}_{l w}$ a razão de mistura de água de chuva ou gelo. A taxa de deposição úmida para uma dada camada de espessura $\Delta z$ é proporcionalmente dada por:

$$
\left(\frac{\partial \overline{\boldsymbol{s}}}{\partial \boldsymbol{t}}\right)_{\substack{\text { dep } \\ \text { umida }}} \propto-\frac{\boldsymbol{k}_{\boldsymbol{H}} \boldsymbol{r}_{\text {lw }} \overline{\boldsymbol{s}}_{\substack{\text { fase } \\ \text { gassa }}} \boldsymbol{p}_{r}}{\Delta \boldsymbol{z}}
$$

onde $\overline{\boldsymbol{s}}_{\text {fase }}$ se refere à razão de mistura da espécie na fase gasosa dentro da porção da nuvem em precipitação, e $\boldsymbol{p}_{\boldsymbol{r}}$ a taxa de precipitação. Os termos $\boldsymbol{r}_{\boldsymbol{t} w}$ e $\boldsymbol{p}_{\boldsymbol{r}}$ podem ser obtidos a partir das parametrizações de microfísica e de cumulus do modelo atmosférico.

\subsection{Transferência radiativa, taxas de aquecimento e de fotólise}

A radiação solar é a energia que governa os processos físicos e químicos na atmosfera terrestre. Ao ser transmitida através da atmosfera ela interage com seus constituintes e aquece a superfície do planeta. Em resposta a este aquecimento, a superfície terrestre emite radiação no infravermelho, a qual é absorvida pelo vapor d'água e outros gases do efeito estufa. A solução da equação de transferência radiativa em modelos atmosféricos tem como objetivo o cálculo das taxas de aquecimento, saldo de radiação na superfície e, no caso de modelos que incluem a reatividade química, de taxas de fotólise.

Em modelos atmosféricos é usual a utilização de uma família de métodos numéricos comumente denominados por Dois Fluxos (em inglês Two-Stream) para a solução da equação de transferência radiativa, considerando uma camada homogênea [Meador e Weaver, 1980]. Esta equação, em termos da radiância I, pode ser escrita como:

$$
\begin{aligned}
& \mu \frac{d \boldsymbol{I}_{\lambda}\left(\tau_{\lambda}, \mu\right)}{\boldsymbol{d} \tau_{\lambda}}=\boldsymbol{I}_{\lambda}\left(\tau_{\lambda}, \mu\right)-\boldsymbol{J}_{\lambda}\left(\tau_{\lambda}, \mu\right) \\
& -\boldsymbol{J}_{0 \lambda}\left(\tau_{\lambda}, \mu, \mu_{0}\right)-\boldsymbol{B}_{0 \lambda}\left(\tau_{\lambda}, \boldsymbol{T}\right)
\end{aligned}
$$

onde $\tau$ é a espessura ótica, $\mu$ é cosseno do ângulo zenital, $\mu_{0}$ é cosseno do ângulo zenital solar e $\boldsymbol{T}$ é a temperatura local. Os termos $\boldsymbol{J}_{\boldsymbol{0}} \boldsymbol{J}_{\boldsymbol{0}} \boldsymbol{e} \boldsymbol{B}_{\boldsymbol{0}}$ representam a função de espalhamento para a radiação difusa, a função fonte para a radiação solar espalhada e a função fonte para a radiação de onda longa emitida, respectivamente, e são dados por:

$$
\begin{aligned}
& \boldsymbol{J}_{\lambda}\left(\tau_{\lambda}, \mu\right)=\frac{\omega_{0}}{2} \int_{-1}^{1} \boldsymbol{I}\left(\tau_{\lambda}, \mu^{\prime}\right) \boldsymbol{P}_{\lambda}\left(\mu, \mu^{\prime}\right) d \mu^{\prime} \\
& \boldsymbol{J}_{0 \lambda}\left(\tau_{\lambda}, \mu, \mu_{0}\right)=\frac{\omega_{0}}{4 \pi} \boldsymbol{P}_{\lambda}\left(\mu, \mu_{0}\right) \boldsymbol{F}_{s} \exp \left(-\frac{\tau_{\lambda}}{\mu_{0}}\right) \\
& \boldsymbol{B}_{0 \lambda}\left(\tau_{\lambda}, \boldsymbol{T}\right)=\left(1-\omega_{0}\right) \boldsymbol{B}_{\lambda}[\boldsymbol{T}]
\end{aligned}
$$

A classe de soluções Dois Fluxos consiste basicamente na divisão da radiância em duas componentes, a ascendente $(+)$ e descendente (-), cada uma delas representada por termos de retro espalhamento e espalhamento frontal nas funções de fase $\boldsymbol{P}_{\lambda}$ da função de espalhamento na Equação 32. Existem na literatura diversos métodos numéricos propostos para a solução da equação de transferência radiativa a partir da premissa de Dois Fluxos, e a escolha do mesmo é sempre um compromisso entre a acurácia desejada, em função do problema a ser resolvido, e a capacidade computacional disponível (Toon et al., 1989). Além disso, os diversos modelos de transferência radiativa diferem entre si em função do tratamento adotado para os processos de absorção de gases, aerossóis e gotas de nuvens. A extinção total e fração devido ao espalhamento, denominada albedo simples de espalhamento $\left(\omega_{0}\right)$ devem incluir a contribuição de gases, aerossóis e gotas de nuvem. 
Em modelos atmosféricos acoplados destinados a estudos de poluição do ar, soluções acuradas para os processos de absorção e espalhamento de gases, aerossóis e nuvens são altamente desejáveis porque podem, dependendo das suas concentrações na atmosfera e propriedades óticas, representarem variações importantes no cálculo das taxas de aquecimento atmosférico e, portanto, erros consideráveis no cálculo da temperatura:

$$
\left(\frac{\partial T}{\partial t}\right)_{\text {rad }}=\frac{1}{c_{p, m}}\left(\frac{d Q_{\text {solar }}}{d t}+\frac{d Q_{i r}}{d t}\right)=\frac{1}{c_{p, m}} \frac{\partial F}{\partial z}
$$

onde $\boldsymbol{F}\left(\mathrm{W} / \mathrm{m}^{2}\right)$ é o fluxo radiativo líquido, somado sobre todos os comprimentos de onda e $\boldsymbol{Q}_{\text {solar }}$ e $\boldsymbol{Q}_{i r}$ são as taxas de aquecimento solar e infravermelho $(\mathrm{J} / \mathrm{kg})$.

Além disso, as taxas de fotólise, usadas na Equação 14, também dependem dentre outras, das condições de nebulosidade e carga de aerossóis na atmosfera e suas propriedades óticas, por afetarem a disponibilidade de fótons. A taxa de fotólise para uma determinada espécie química A é calculada partindo da quantidade de fótons (por comprimento de onda $\lambda$ ) disponíveis numa dada seção da atmosfera (o fluxo acitínico, $\mathrm{F}_{\lambda}$ ), do coeficiente de absorção da molécula $\boldsymbol{A}\left(\sigma_{A}, \lambda\right)$ (que fornece a probabilidade de absorção de fótons de dado $\lambda$ pela molécula), da probabilidade de uma absorção em $\lambda$ ser seguida de uma dissociação (o quantum yield da reação, $\phi_{A}, \lambda$ ) e, integrando o produto destas três quantidades em todo o espectro $\lambda$, ou seja:

$$
j_{A}=\int \sigma_{A \lambda} \phi_{\lambda} F_{\lambda} d \lambda
$$

Em condições extremas de poluição, como é freqüente na Amazônia e Brasil Central durante a estação de queimadas, a consideração da presença de aerossol pode chegar a representar uma atenuação de 10\%-20\% na radiação solar instantânea na superfície terrestre. Modelos atmosféricos que incluem este efeito respondem com modificações significativas nos perfis de temperatura, umidade relativa e balanços de calor sensível e calor latente. As taxas de fotólise calculadas, por sua vez, podem variar em até $70 \%$, em função da consideração do efeito radiativo do aerossol, e representar uma superestimativa do ozônio troposférico que pode chegar a 300\% (Albuquerque et al., 2006). Assim, a inclusão adequada e consistente dos processos de espalhamento e absorção dos diversos constituintes atmosféricos, através do acoplamento do esquema de radiação com as parametrizações de microfísica de nuvens e de aerossóis é necessária.

Neste trabalho, não se tratou de processos de microfísica de aerossóis, por exemplo, coagulação e de reatividade em meios com múltiplas fases de água que levam a termos adicionais na equação da continuidade (Equação 3).

\section{RESULTADOS COM O SISTEMA CATT- BRAMS}

O Centro de Previsão de Tempo e Estudos Climáticos (INPE/CPTEC) tem avançado na direção do desenvolvimento de modelos acoplados com aerossóis e traçadores, como o sistema 'Coupled Aerosol and Tracers Transport model to the Brazilian developments on the Regional Atmospheric Modeling System' (CATT-BRAMS, Freitas et al., 2005, 2006, 2007A, B, Longo et al., 2006, 2007) e, recentemente, com reatividade química (CATT-Chemistry, Longo et al., 2009, em preparação). Tratase de um modelo numérico de emissão, transporte e deposição de aerossóis e traçadores atmosféricos acoplado ao modelo BRAMS (www.cptec.inpe.br/brams) e com o efeito radiativo de aerossóis de queimadas. O CATT é um modelo on-line 3D Euleriano, que prognostica a concentração de contaminantes atmosféricos de forma simultânea e totalmente consistente com o estado atmosférico simulado pelo BRAMS. A Figura 6 ilustra os principais processos envolvidos na emissão, transporte e deposição de gases e aerossóis e que são simulados pelo sistema

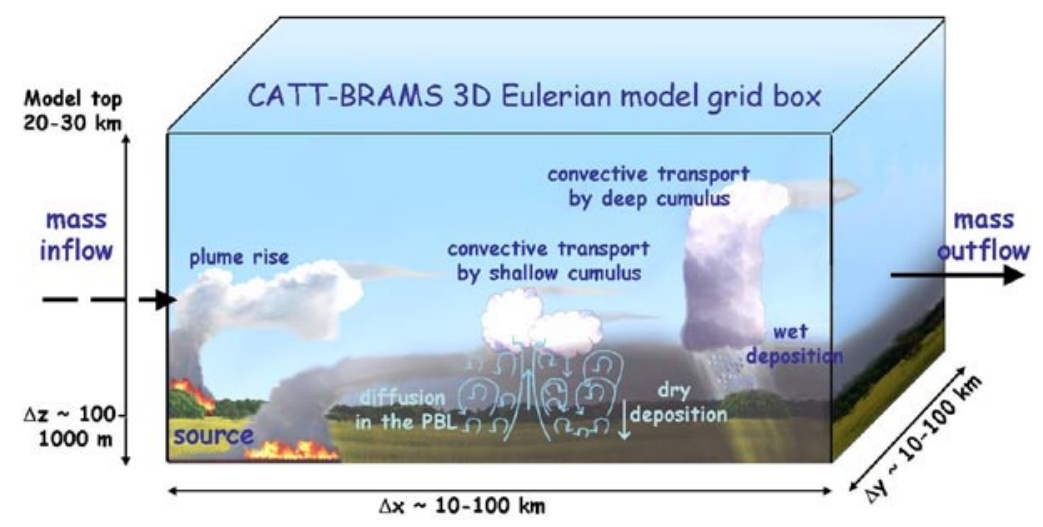

Figura 6 - Diagrama descrevendo os principais processos sub-grade relevantes para modelagem da poluição do ar e incluídos no sistema CATTBRAMS. 
CATT-BRAMS. Este sistema de modelagem foi validado utilizando dados observacionais em superfície e na baixa troposfera (medidas em avião instrumentado) e dados obtidos por sensores remotos durante a estação seca de 2002 (Freitas et al., 2007A, B e Longo et al., 2007). Os resultados demonstraram que o modelo possui emissão e deposição realísticas, e dinâmica robusta (não somente no que diz respeito à advecção na escala da grade, mas também aos processos convectivos de transporte em escala de sub-grade, difusão na CLP e mecanismo de plume rise de emissões quentes) para gases relativamente inertes, como o CO, e para aerossóis de queimadas PM2.5.

O sistema CATT-BRAMS tem sido utilizado operacionalmente no CPTEC para a previsão de qualidade do ar para monóxido de carbono e material particulado desde 2003 (veja meioambiente.cptec.inpe.br). Um exemplo do desempenho deste sistema pode ser visualizado na Figura 7. Em abril de 2008, agricultores argentinos realizaram diversas queimadas para conversão de florestas em pastagem, produzindo enorme pluma de fumaça (Figura 7B, imagem do MODIS do dia 17/04/2008) que atingiu Buenos Aires degradando a qualidade do ar local (Figura 7C) e a visibilidade. A Figura 7A mostra a previsão em tempo real (iniciada às 00UTC 16/04/2008) para as 03UTC 17/04/2008 da pluma de fumaça invadindo a região de Buenos Aires produzida pelo CATT-BRAMS, demonstrando a capacidade do sistema em capturar, a partir de produto de sensoriamento remoto de fogo, as emissões, realizar o transporte e prever concentrações de CO e PM2.5.

Alguns resultados recentes com reatividade química são mostrados e discutidos a seguir nesta seção. Uma simulação numérica para a estação seca da Amazônia de 2002 foi realizada com uma grade com $50 \mathrm{~km}$ de espaçamento de grade horizontal

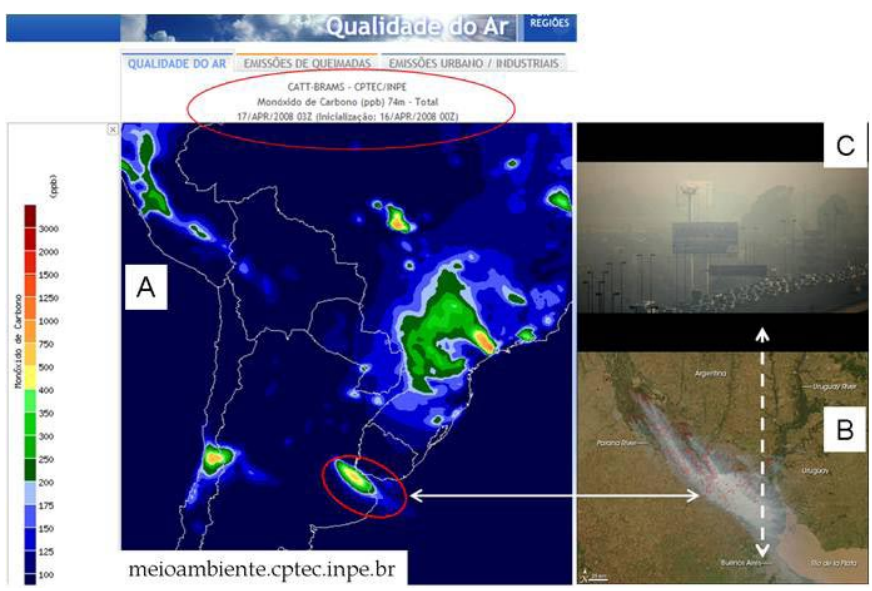

Figura 7 - (A) Previsão em tempo real de monóxido de carbono para às 03UTC 17/04/2008, iniciada às 00UTC 16/04/2008, do sistema CATT-BRAMS do CPTEC/INPE (http://meioambiente.cptec.inpe.br). (B) Imagem no visível do MODIS em 17/04/2008. (C) foto da cidade de Buenos Aires em 17/04/2008. e espaçamento de grade vertical variável entre 150 e 850 metros. Dados de análise do modelo global do CPTEC T126 foram utilizados como condição inicial e de contorno atmosférica. A condição inicial da umidade do solo foi obtida através de Gevaerd e Freitas (2006). O período de integração foi de 105 dias, iniciando-se às 00UTC de 15/07/2002. O mecanismo químico utilizado para a reatividade foi o RACM (Stockwell et al., 1997) pré-processado pelo sistema Simplified Preprocessor for Atmospheric Chemical Kinetics (SPACK, Djouad et al., 2002, Longo et al., 2009). O RACM possui 72 espécies, sendo 36 transportáveis, e 237 reações cinéticas e de fotólise. Nesta simulação, as reações de fotólises foram obtidas por meio de valores tabelados, produzidos pelo SPACK, em função do ângulo zenital, e somente a fase gasosa foi considerada. As condições iniciais para as espécies transportáveis se referem a uma condição de atmosfera não perturbada e horizontalmente homogênea. Emissões de origem antrópica (industrial, transporte, urbana) do inventário RETRO foram interpoladas para a grade do modelo utilizando um pré-processador de emissões; o mesmo para as emissões biogênicas do programa GEIA. Emissões de queimadas foram processadas em cada ponto de grade utilizando o modelo de emissões Brazilian Biomass Burning Emission Model (3BEM, Freitas et al., 2005, Longo et al., 2007) e focos de queimadas derivados de diversos sensores a bordo de satélites. Um período de 15 dias (15 a 30 de julho) foi utilizado para produzir um estado de composição química da atmosfera mais realístico, partindo da condição não perturbada, conforme referido anteriormente, até um estado modificado pelas emissões. A condição de contorno lateral constante para fluxo entrando e variável com fluxo saindo do domínio foram utilizadas na integração. $\mathrm{O}$ mecanismo químico foi resolvido com o método de Rosenbrock de $2^{\mathrm{a}}$ ordem (Verwer et al. 1997), porém com passo de tempo de integração adaptativo, o que permite maior estabilidade e eficiência numéricas.

A Figura 8 apresenta um exemplo de resultados obtidos desta simulação. O painel (A) mostra PM2.5 integrado verticalmente na coluna $\left(\mathrm{em} \mathrm{mg} \mathrm{m}^{-2}\right.$ ) às $18 \mathrm{UTC}$ de 27/08/2002, exibindo uma grande pluma de escala continental atravessando a América do Sul, sendo este transporte de longa distância de aerossóis de queimadas intensificado por jatos de baixos níveis ocorrendo na costa leste da Cordilheira dos Andes. O transporte concomitante de vapor d'água da região Amazônica ( $\mathrm{g} / \mathrm{kg}$, a $1400 \mathrm{~m}$ acima da superfície) é representado no painel (B) e alimenta complexos convectivos precipitantes ocorrendo a sudeste da Argentina (vide o painel (C)). Os painéis (D) e (E) mostram o transporte vertical, na alta troposfera $(10,7 \mathrm{~km})$, de $\mathrm{CO}$, o qual atua aproximadamente como um traçador inerte, e a deposição úmida de PM2.5 através da remoção de material particulado entranhado na nuvem pela precipitação sobre a superfície, respectivamente. 
$\mathrm{O}_{3}$ foi uma das espécies reativas simuladas pelo mecanismo com máximos em superfície ocorrendo próximo às regiões emissoras de precursores (região de queimadas e megacidades) e ao longo dos corredores de exportação destes. A Figura 9 apresenta uma comparação entre dois modelos. $\mathrm{O}$ painel (B) apresenta a média mensal para setembro do $\mathrm{O}_{3}$ em superfície simulada pelo modelo global GEOS-CHEM (Bey et al., 2001) numa resolução espacial de 4 x 4 graus. O máximo de $\mathrm{O}_{3}$ é simulado na região central da América do Sul, correspondendo à região de maior número de focos de queimadas. Os valores típicos simulados na região perturbada estão entre 30 e 60 ppbv. $\mathrm{O} \mathrm{O}_{3}$ simulado com a versão do CATT-BRAMS, incluindo reatividade química, apresenta características bastante semelhantes em termos dos padrões de distribuição espacial desta espécie e valores máximos entre 30 e 65 ppbv. Apesar das duas simulações terem resoluções bastante diferentes, a média temporal de 1 mês remove transientes e permite uma comparação consistente. Obviamente, uma resolução mais fina permite maior detalhamento e resolve melhor processos locais e de menor escala, sendo, no entanto suavizados pela média temporal. A Figura 10 introduz um balanço da deposição seca de $\mathrm{O}_{3}$ ao longo de 2 meses de simulação (agosto e setembro). Trata-se do total acumulado em superfície em unidade $\mathrm{g} / \mathrm{m}^{2}$. $\mathrm{O} \mathrm{O}_{3}$ se deposita com relativa eficiência e, conforme descrito anteriormente, se forma tipicamente quando precursores emitidos por combustão $\left(\mathrm{NO}_{\mathrm{x}}\right)$ são transportados para uma região permeada de COV e na presença de radiação solar. Na Figura 10, esta situação parece estar bem representada com 3 corredores de deposição de $\mathrm{O}_{3}$. Um é formado ao longo do Estado de São Paulo, partindo de sua capital e região do entorno, com suas emissões de precursores de origem urbana (por exemplo, $\mathrm{NO}_{\mathrm{X}}$ ) sendo transportados continente adentro pelo vento predominante de sudeste. Neste caso, o $\mathrm{O}_{3}$ se forma e se deposita a partir, principalmente, das reações envolvendo o $\mathrm{NO}_{\mathrm{X}}$ com os $\mathrm{COV}$ das áreas rurais. Dois outros corredores ao longo da Cordilheira dos Andes, também são evidentes nesta figura, porém a origem dos precursores neste caso envolve principalmente emissões de queimadas na região contornada em preto. Um dos corredores se alinha para o sul na costa leste desta cadeia de montanhas, e parece estar relacionado com jatos de baixos níveis, transportando precursores, bem como o próprio $\mathrm{O}_{3}$ formado vento acima, e gerando uma linha de deposição ao longo desta cadeia. Um terceiro corredor ao norte, também seguindo os Andes, aparece neste resultado e está associado à entrada de frentes frias e ao deslocamento para o sul do centro do anticiclone sobre o Atlântico Sul. Nestes casos, surge uma componente de sudeste atuando na região de queimadas transportando emissões em direção a parte noroeste da Amazônia. Esta área, predominantemente de florestas, constitui uma região bastante propícia para a formação de $\mathrm{O}_{3}$ pela alta taxa de $\mathrm{COV}$ de origem biogênica presente na atmosfera. Como o $\mathrm{O}_{3}$ contribui para a degradação de plantas, a existência destes corredores coloca questões sobre a magnitude

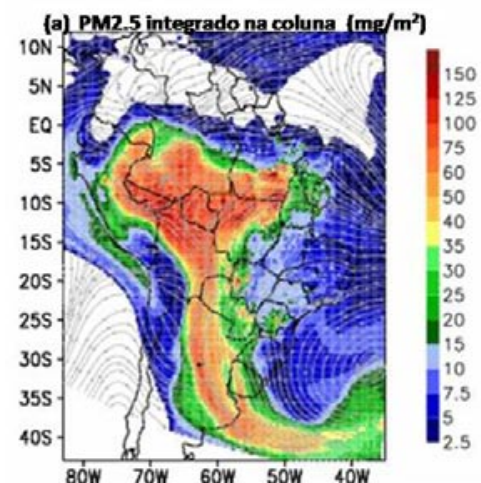

\section{Tempo: 18 UTC 27/08/2002}
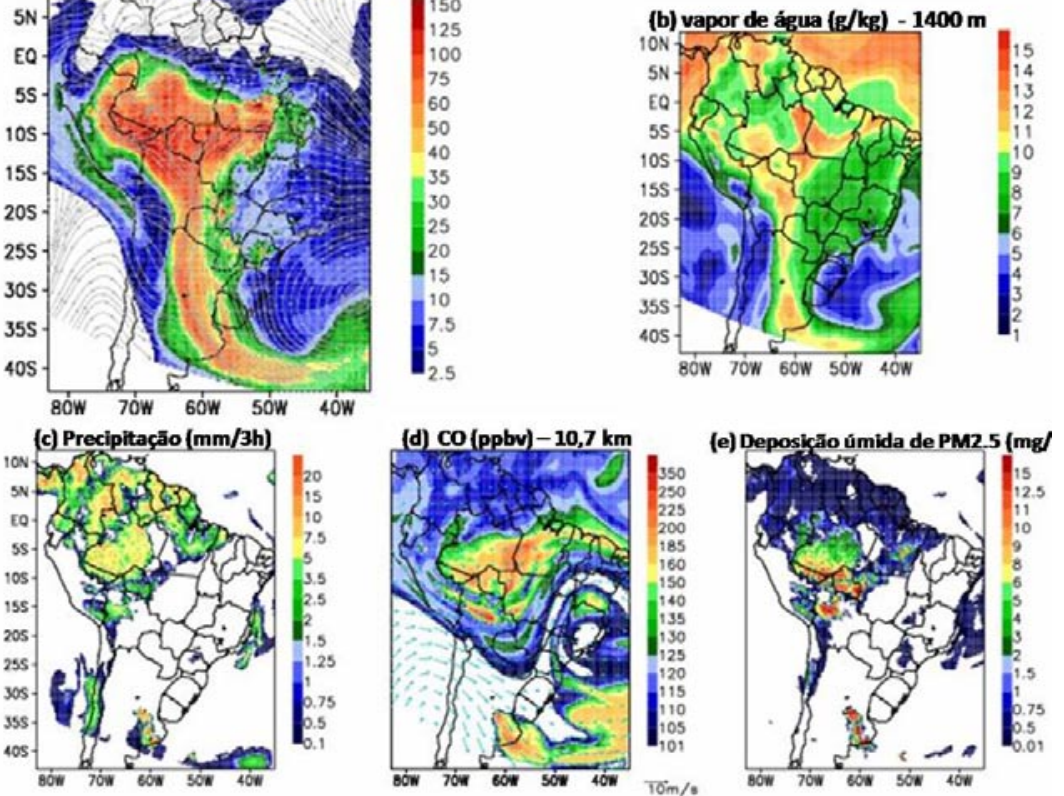

Figura 8 - Exemplo de resultado de simulação do CATT-BRAMS para as 18UTC de 27/08/2002. (A) PM2.5 verticalmente integrado na coluna (mg $\mathrm{m}$-2) mostrando o transporte a longa distância de aerossóis de queimadas por jatos de baixos níveis. (B) o mesmo transporte para o vapor d'água em 1400 metros acima da superfície. (C) simulação da precipitação acumulada em 3 horas. (D) CO transportado para a troposfera (10.7 km) por sistemas convectivos profundos e precipitantes. (E) deposição úmida de PM2.5. 
do efeito deletério desta deposição sobre florestas e plantações (como exemplos, de soja no Mato Grosso, e de cana-de-açúcar em São Paulo) nestes locais.

Um balanço de dois meses de deposição de PM2.5 de queimadas é apresentado na Figura 11. O painel (A) mostra a deposição seca (em $10^{-3} \mathrm{~g} \mathrm{~m}^{-2}$ ) indicando que os máximos ocorrem localmente, isto é, próximo a região emissora. O PM2.5 possui sedimentação gravitacional desprezível, se deslocando na atmosfera com praticamente a mesma velocidade vertical das massas de ar. Assim, a maior parte do material depositado corresponde a emissões em superfície, principalmente na fase de combustão fria (smoldering) ou trazidas para níveis próximos à superfície por transporte de material originalmente presente em níveis mais altos. A deposição úmida, porém apresenta dois máximos (painel $\mathrm{B}$, em $\mathrm{g} \mathrm{m}^{-2}$ ): um local, próximo às fontes porém mais a oeste, associado à precipitação local sobre a Amazônia Legal e outro distante e ao sul. Este segundo máximo está predominantemente associado ao transporte a longa distância e na baixa troposfera ( $\sim 2 \mathrm{~km}$ de altura acima da superfície) de PM2.5 por jatos de baixos níveis e/ou pelo ramo oeste do anticiclone do Atlântico Sul. Este transporte traz embutido vapor d'água da Amazônia (veja Figura 8 b) que predispõe à formação de sistemas convectivos precipitantes no sul e sudeste da América do Sul, os quais promovem a deposição de grande parte do material particulado fino carreado da região de queima. Uma questão relevante que surge deste processo é se este constitui um importante ciclo biogeoquímico conectando a Amazônia com a parte Sul da América do Sul.

O efeito dos aerossóis de queimadas na precipitação, do ponto de vista da estabilização termodinâmica provocada pela
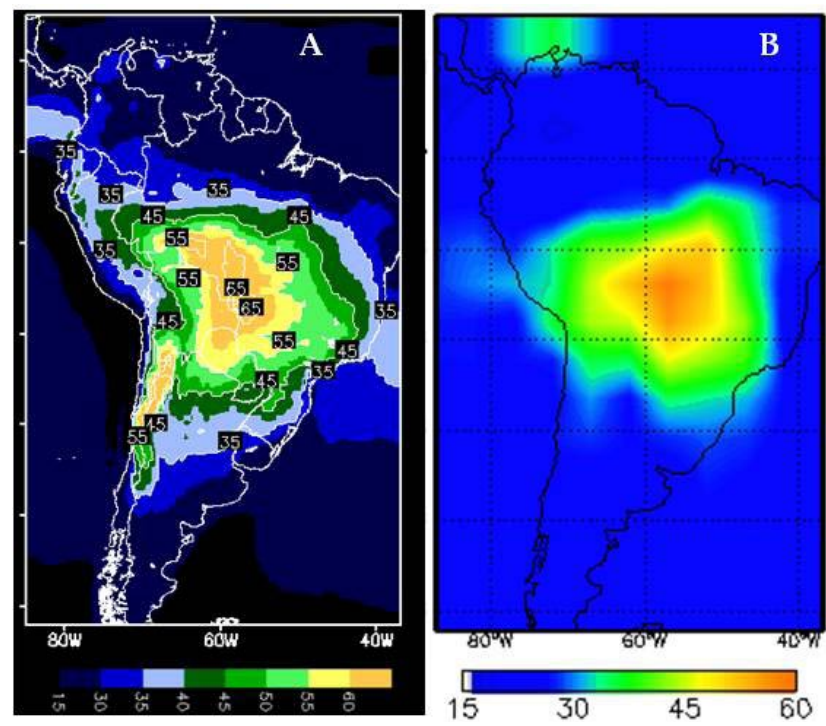

Figura 9 - Ozônio em superfície (ppbv) média mensal para o mês de setembro, (A) modelo CCATT-BRAMS com resolução de 50 x $50 \mathrm{~km}$ e (B) modelo GEOS-CHEM com resolução de 4 x 4 graus. absorção e espalhamento de onda curta em níveis mais altos e a conseqüente redução da radiação disponível em superfície, é discutido nesta seção. Para entender o efeito direto dos aerossóis, uma segunda simulação foi realizada na qual estes não são incluídos no cálculo da transferência radiativa. Neste estudo as propriedades ópticas dos aerossóis consideradas na transferência radiativa são baseadas em uma climatologia das observações da rede AERONET na região Amazônica e, portanto, intrinsecamente contêm a informação da composição química e distribuição de tamanho dos aerossóis (Procópio et al., 2003). A Figura 12 apresenta o resultado, com enfoque na região de Rondônia, com alta persistência de níveis de aerossóis provenientes de queimadas. São mostradas duas séries temporais de precipitação simulada ( $\mathrm{mm} / 3 \mathrm{~h}$, média espacial no quadrado vermelho por 3 meses) para os dois estudos. Comparando as duas séries, evidencia-se o efeito dos aerossóis com uma sensível diferença nas taxas horárias, principalmente no período de pico das queimadas (agosto e setembro). O efeito final aparece na linha contínua, com um total de aproximadamente $300 \mathrm{~mm}$ acumulados no caso sem aerossol e de $200 \mathrm{~mm}$ com aerossol. Assim, só o efeito direto dos aerossóis produziu uma redução da ordem de $30 \%$ na precipitação acumulada. Observando os mapas de climatologia (à esquerda e em baixo) e precipitação observada (à direita e em baixo) desta figura, depreende-se que o modelo consegue simular coerentemente os valores de precipitação típicos da região estudada. Além disto, a redução prevista pela simulação com os aerossóis parece estar representada no dado de precipitação observada. No entanto, a falta de dados com maior densidade espacial e séries mais longas não permite uma conclusão segura de que se trata do

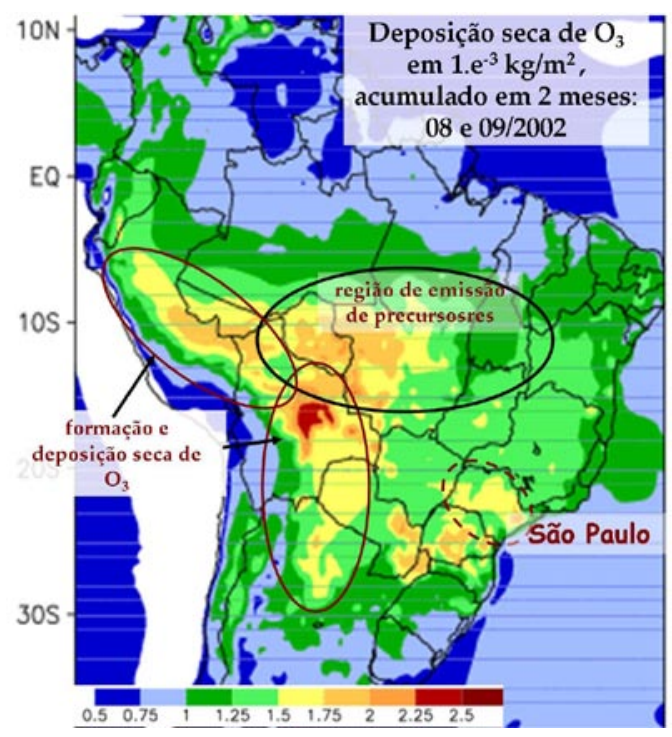

Figura 10 - Deposição seca de ozônio acumulado em dois meses (agosto e setembro de 2002). 


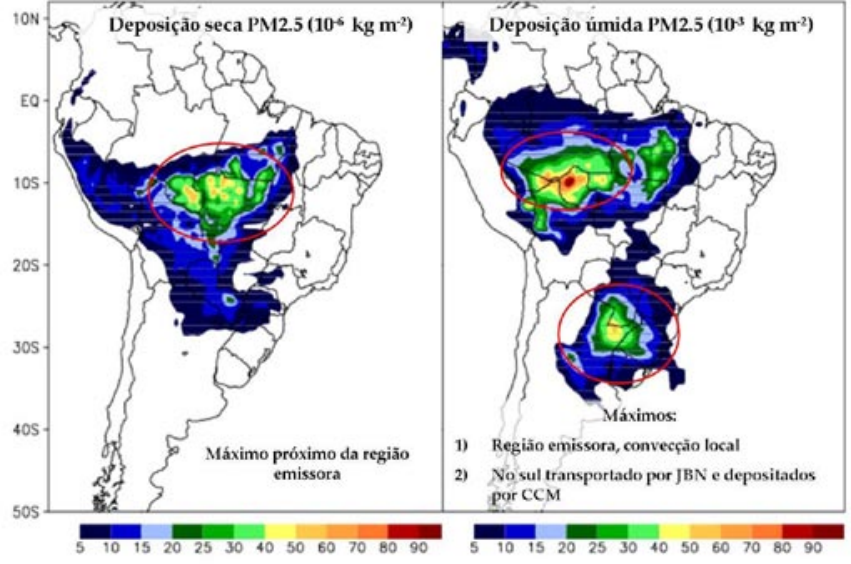

Figura 11 - Deposição seca e úmida de PM2.5 acumulado em dois meses (agosto e setembro de 2002). JBN e CCM significam jatos de baixos níveis e complexos convectivos de mesoescala, respectivamente.

efeito direto dos aerossóis ou uma variabilidade natural do clima local. Uma avaliação geral sobre a América do Sul indica que, em regiões com alta persistência de aerossóis de queimadas, a redução da precipitação acumulada em três meses fica acima de $75 \mathrm{~mm}$. Aparecem também efeitos a longa distância associados ao transporte de aerossóis, como na região situada no norte de Argentina. Esta região é normalmente palco de ocorrência de complexos convectivos de mesoescala e a presença dos aerossóis vindos da Amazônia influem na formação destes sistemas. Como a convecção interage na circulação atmosférica de uma forma altamente não linear, a perturbação causada pelos aerossóis pode afetar também regiões mais distantes através das chamadas teleconexões. Além disso, uma avaliação conclusa sobre o impacto dos aerossóis na atmosfera requer também, o desenvolvimento de parametrizações que prognostiquem água de chuva em termos da quantidade de CCN na base da nuvem e inclusão na parametrização convectiva. Mais complexo ainda, porém não menos importante do ponto de vista do balanço de radiação, é necessário que os conjuntos de propriedades ópticas de nuvens na transferência radiativa sejam coerentes com os impactos prognosticados dos aerossóis nas nuvens.

\section{CONCLUSÕES}

Este trabalho apresenta uma breve descrição geral do tema de modelagem da atmosfera incluindo emissão, transporte, transformação e deposição de gases traço e aerossóis. Dentre os componentes da equação da continuidade, o termo de reatividade química se destaca pela complexidade de problema em si, na obtenção da solução numérica e no alto custo computacional envolvido. Por outro lado, o papel dos aerossóis de queimadas

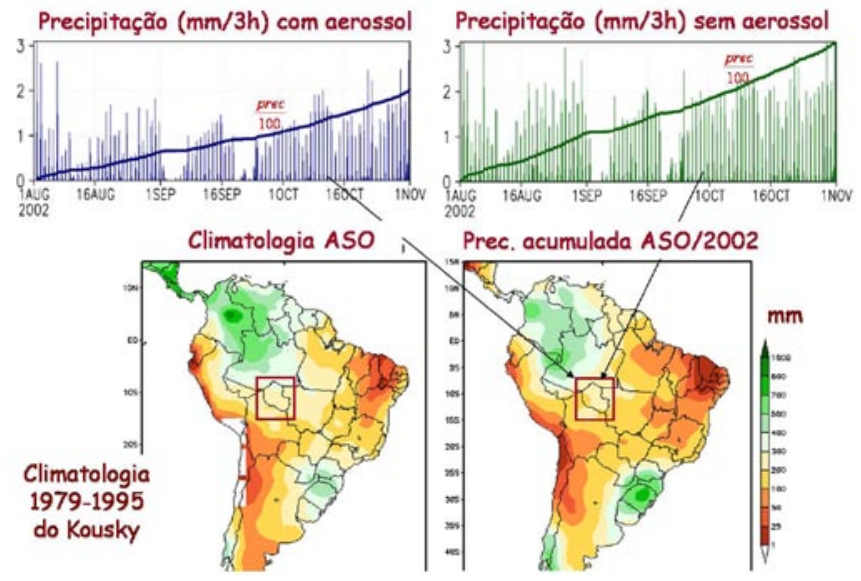

Figura 12 - Efeito direto dos aerossóis de queimadas na precipitação enfocando a região de Rondônia.

na modificação do balanço radiativo e conseqüente impacto na formação de sistema convectivos, constitui ainda um problema em aberto. Trabalhos futuros incluirão o efeito indireto através da alteração da densidade de núcleos de condensação de nuvens e, assim, o papel dos aerossóis de queimadas poderão ser melhor quantificados.

Estes modelos não constituem os denominados modelos ambientais ou do sistema terrestre, porem constituem parte essencial destes. Sendo necessários não somente para responder às questões das mudanças da composição química da atmosfera e os impactos na qualidade do ar, mas também para as questões maiores envolvendo mudanças globais, além de permitirem projeções de cenários futuros com maior confiabilidade para tomada de decisões pela sociedade civil e organismos governamentais.

\section{AGRADECIMENTOS}

Este trabalho foi realizado com suporte parcial das seguintes agências e projetos: Inter-American Institute for Global Change Research (IAI) CRN II 2017 e US National Science Foundation (Grant GEO-0452325); NASA Headquarters (NRA-03-OES-02, NRA-97-MTPE-02 e NRA-02-OES-06), CNPq (305059/2005-0, 309922/2007-0) e FAPESP (04/134451, 01/14981-6).

\section{REFERÊNCIAS}

ALBUQUERQUE, L., K. LONGO, S. R. FREITAS, T. TARASOVA, A. PLANA FATTORI, C. NOBRE, L. V. GATTI. Sensitivity studies on the photolysis rates calculation 
in Amazonian atmospheric chemistry - Part I: The impact of the direct radiative effect of biomass burning aerosol particles. Atmospheric Chemistry and Physics Discussions, v. 5, p. 9325-9353, 2005.

ANDREAE, M. O., Biomass burning: Its history, use and distribution and its impact on environmental quality and global climate, in Global Biomass Burning: Atmospheric, Climatic and Biospheric Implications, editado por J. S. Levine, pp. 3-21, MIT Press, Cambridge, Mass., 1991.

ANDREAE, M., AND MERLET, P.: Emission of trace gases and aerosols from biomass burning, Global Biogeochemical Cycles, 15, 4, 955-966, 2001.

ANDREAE M. O., D. ROSENFELD, P. ARTAXO, A. A. COSTA, G. P. FRANK, K. M. LONGO, AND M. A. F. SILVA-DIAS. Smoking rain clouds over the Amazon, Science, 303, 1337-1342., 2004.

ANDREAE, M. O., C. D. JONES E P. M. COX. Strong presentday aerosol cooling implies a hot future, Nature, V. 435, 30, pp. 1187-1190, 2005.

ARAKAWA, A., AND W. H. SCHUBERT. Interaction of a cumulus cloud ensemble with the large scale environment, Part I, J. Atmos. Sci., 31, 674- 701, 1974.

ARTAXO,P.,J.V.MARTINS,M.A.YAMASOE,A.S.PROCÓPIO, T. M. PAULIQUEVIS, M. O. ANDREAE, P. GUYON, L. V. GATTI, A.M. CORDOVA LEAL. Physical and chemical properties of aerosols in the wet and dry season in Rondônia, Amazonia. J. Geophys. Res. 107 (D20): 49.1-49.14, 2002.

BEY I., D. J. JACOB, R. M. YANTOSCA, J. A. LOGAN, B. FIELD, A. M. FIORE, Q. LI, H. LIU, L. J. MICKLEY, AND M. SCHULTZ. Global modeling of tropospheric chemistry with assimilated meteorology: Model description and evaluation, J. Geophys. Res., 106, 23,073-23,096, 2001.

CHATFIELD, R. B., AND DELANY, A. C.: Convection links biomass burning to increased tropical ozone: However, models will tend to overpredict O3, J. Geophys. Res., 95, 18,473-18,488, 1990.

COX, P. M. et al. Increasing risk of Amazonian drought due to decreasing aerosol pollution. Vol 453, Nature, 8 May 2008.

DJOUAD, R., SPORTISSE, B., AUDIFFREN, N. Numerical simulation of aqueous-phase atmospheric models: use of a non-autonomous Rosenbrock method. Atmospheric Environment, vol. 36- 5, 873-879(7), 2002.

FREITAS, S. R., K. LONGO, M. DIAS, R. CHATFIELD, P. DIAS, P. ARTAXO, M. ANDREAE, G. GRELL, L. RODRIGUES, A. FAZENDA AND J. PANETTA. The Coupled Aerosol and Tracer Transport model to the Brazilian developments on the Regional Atmospheric Modeling System (CATT-BRAMS). Part 1: Model description and evaluation. Atmos. Chem. Phys. Discuss., 7., 8525-8569, 2007.
FREITAS, S. R., K. M. LONGO, R. CHATFIELD, D. LATHAM, M. A. F. SILVA DIAS, M. O. ANDREAE, E. PRINS, J. C. SANTOS, R. GIELOW AND J. A. CARVALHO JR. Including the sub-grid scale plume rise of vegetation fires in low resolution atmospheric transport models. Atmospheric Chemistry and Physics, v. 7, p. 3385-3398, 2007.

FREITAS, S. R., LONGO, K. M., M. ANDREAE. Impact of including the plume rise of vegetation fires in numerical simulations of associated atmospheric pollutants. Geophys. Res. Lett., 33, L17808, doi:10.1029/2006GL026608, 2006.

FREITAS, S., K. LONGO, M. SILVA DIAS, P. SILVA DIAS, R. CHATFIELD, E. PRINS, P. ARTAXO, G. GRELL AND F. RECUERO. Monitoring the transport of biomass burning emissions in South America, Environmental Fluid Mechanics, DOI: 10.1007/s10652-005-0243-7, 5 (1-2), p. $135-167,2005$.

FREITAS, S. R. Modelagem Numérica do Transporte e das Emissões de Gases Traço e Aerossóis de Queimadas no Cerrado e Floresta Tropical da América do Sul. Tese de Doutoramento em Física Aplicada - Instituto de Física, Universidade de São Paulo, agosto de 1999. 204 p.

FROMM, M., ALFRED, J., HOPPEL, K., HORNSTEIN, J., BEVILACQUA, R., SHETTLE, E., SERVRANCKX, R., LI, Z., AND STOCKS, B. Observations of boreal forest fire smoke in the stratosphere by POAM III, SAGE II, and lidar in 1998, Geophys. Res. Lett., 27, 1407--1410, 2000.

GEVAERD, R. e S. R. FREITAS. Estimativa operacional da umidade do solo para iniciação de modelos de previsão numérica da atmosfera. Parte I: Descrição da metodologia e validação. Revista Brasileira de Meteorologia, 21, 3a, 59-73, 2006.

GRELL, G. Prognostic evaluation of assumptions used by cumulus parameterizations, Mon. Wea. Rev., 121, 764-787, 1993.

GUENTHER,A., B. BAUGH, G. BRASSEUR, J. GREENBERG, P. HARLEY, L. KLINGER, D. SERÇA, AND L. VIERLING. Isoprene emission estimates and uncertainties for the Central African EXPRESSO study domain, J. Geophys. Res., 104(D23), 30,625-30,639, 1999.

HAIRER, E. AND WANNER, G. Solving Ordinary Differential Equations II. Stiff and Differential-Algebraic Problems, Springer-Verlag, Berlin, 1991.

ICHOKU, C., AND KAUFMAN, Y.J. A method to derive smoke emission rates from MODIS fire radiative energy measurements. IEEE Transactions on Geoscience and Remote Sensing, 43, 11, 2636- 2649, 2005.

IPCC: Climate Change 2007: The Physical Science Basis. Contribution of Working Group I to the Fourth Assessment Report of the Intergovernmental Panel on Climate Change 
[Solomon, S., D. Qin, M. Manning, Z. Chen, M. Marquis, K.B. Averyt, M. Tignor and H.L. Miller (eds.)]. Cambridge University Press, Cambridge, United Kingdom and New York, NY, USA, 996 pp., 2007.

JACOBSON, M. Z. Strong Radiative Heating due to the Mixing State of Black Carbon in Atmospheric Aerosols. Nature, vol. 409, 695-697, 2001.

KAUFMAN, Y. J. Remote Sensing of Direct and Indirect Aerosol Forcing. In: Aerosol Forcing of Climate. Ed. by R. J. Charlson and J. Heintzenberg, John Wiley \& Sons Ltd., 1995.

Koren I., Y. Kaufman, L. A. Remer, J. V. Martins: 2004, Measurement of the Effect of Amazon Smoke on Inhbition of Cloud Formation, Science, 303, 1342-1345.

KUNDERT, S. K. Sparse matrix techniques. In Circuit Analysis, Simulation and Design, Albert Ruehli (editor). North-Holland, 1986.

KUNDERT, S. K.; and SANGIOVANNI-VINCENTELLI, A. In Sparse User's Guide - A Sparse Linear Equation Solver. Version 1.3a. Berkeley. 1988. Obtido de: http://www.netlib. org/sparse/spdoc em 18 Março 2008.

LANSER, D., J.G. VERWER. Analysis of operator splitting for advection-diffusion-reaction problems from air pollution modeling, CWI Report MAS-R9805, 1998.

LONGO, K. M., FREITAS, S. R., SILVA DIAS, M.A.F. DIAS, P. SILVADIAS. Numerical modeling developments towards a system suitable to a real time air quality forecast and climate changes studies in South America, Newsletter of the International Global Atmospheric Chemistry Project, Taiwan, v. 33, p. 12-16, 2006.

LONGO, K., S. R. FREITAS, A. SETZER, E. PRINS, P. ARTAXO, AND M. ANDREAE. The Coupled Aerosol and Tracer Transport model to the Brazilian developments on the Regional Atmospheric Modeling System (CATT-BRAMS). Part 2: Model sensitivity to the biomass burning inventories. Atmos. Chem. Phys. Discuss., 8571-8595, 2007.

LONGO, K., et al. The Coupled Chemistry-Aerosol-Tracer Transport model to the Brazilian developments on the Regional Atmospheric Modeling System (CCATT-BRAMS). Em preparação, 2009.

MEADOR, W. E. e WEAVER. Two-stream approximations to radiative transfer in planetary atmospheres: A unified description of existing methods and a new improvement, J. Atmos. Sci., 37, 630-643, 1980.

MARENGO, J. A., et al. The drought of Amazonia in 2005. J. Clim. 21, 495-516, 2008.

PEREIRA, G. O uso de satélites ambientais para a estimativa dos fluxos de gases traço e de aerossóis liberados na queima de biomassa e sua assimilação em modelos numéricos de qualidade do ar, Tese de Mestrado em Sensoriamento Remoto, INPE, 2008.
POPPE, D., KOPPMANN, R., AND RUDOLPH, J. Ozone formation in biomass burning plumes: Influence of atmospheric dilution, Geophys. Res. Lett., 25, 3823--3826, 1998.

PRINS, E., FELTZ, J., MENZEL, W., AND WARD, D. An overview of GOES-8 diurnal fire and smoke results for SCAR-B and 1995 fire season in South America, J. Geophys. Res., 103, D24, 31,821-31,835, 1998.

PROCÓPIO, A. S., REMER, L. A., ARTAXO, P., KAUFMAN, Y. J., HOLBEN, B. N. 2003. Modeled spectral optical properties for smoke aerosols in Amazonia. Geophysical Research Letters, 30(24): 2.265-2.270. doi: 10.1029/2003GL018063.

PRUPPACHER, H. R., J. D. KLETT. Microphysics of Clouds and Precipitation, second edition, Kluwer Academic Publishers, Dordrecht, The Netherlands, 954 pp., 1997.

ROSENFELD, D. TRMM observed first direct evidence of smoke from forest fires inhibiting rainfall, Geophys. Res. Lett. 26, 20, 3101, 1999.

SAATCHI, S. S.; HOUGHTON, R. A.; DOS SANTOS ALVALÁ, R. C.; SOARES, J. V.; YU, Y. Distribution of aboveground live biomass in the Amazon basin. Global Change Biology, 13, 816-837, 2007.

STOCKWELL W. R.; KIRCHNER F.; KUHN M.; SEEFELD S. A new mechanism for regional atmospheric chemistry modeling. J. Geophys. Res., vol. 102, noD22, pp. 2584725879, 1997.

STULL, R. B. An introduction to boundary layer meteorology. Kluwer Academic Publishers, Norwell, MA. 666 pp, 1988.

TREMBACK, C., POWELL, J., COTTON, W., AND PIELKE, R. The forward in time upstream advection scheme: Extension to higher orders, Mon. Wea. Rev., 115, 540-555, 1987.

VERWER, J. G., et al. A second order Rosenbrock method applied to photochemical dispersion models, MAS-R9717, 1997.

TOON, O. B., C. P. MCKAY, e T. P. ACKERMAN. Rapid calculation of radiative heating rates and photodissociation rates in inhomogeneous multiple scattering atmospheres, J. Geophys. Res., vol. 94, no. D13, 16287-16301, 1989.

TURQUETY, S., LOGAN, J., JACOB, D., HUDMAN, R., LEUNG, F., HEALD, C., YANTOSCA, R., WU, S., EMMONS, L., EDWARDS, D., AND SACHSE, G. Inventory of boreal fire emissions for North America in 2004: the importance of peat burning and pyro-convective injection, J. Geophys. Res., 112, D12S03, doi:10.1029/ 2006JD007281, 2007. 